DESY-97-014

February 1997

\title{
QCD corrections to the longitudinally polarized Drell-Yan process
}

\author{
T. Gehrmann \\ DESY, Theory Group, D-22603 Hamburg, Germany
}

\begin{abstract}
In this paper we calculate the $\mathcal{O}\left(\alpha_{s}\right)$ corrections to the $x_{F}$ - and $y$-distributions of lepton pairs produced in collisions of longitudinally polarized hadrons. The numerical importance of these corrections is studied and consequences for the extraction of the polarized sea quark distributions from a measurement of the longitudinally polarized Drell-Yan cross section are discussed.
\end{abstract}

PACS: 13.88.+e;13.75.Cs;13.85.Qk;12.38.Bx

Keywords: Lepton pair production, polarization, hadron-hadron collisions. 


\section{Introduction}

The production of lepton pairs in hadron collisions, the Drell-Yan process [1], is one of the most powerful tools to probe the structure of hadrons. Its parton model interpretation is straightforward - the process is induced by the annihilation of a quark-antiquark pair into a virtual photon which subsequently decays into a lepton pair. The Drell-Yan process in proton-proton or proton-nucleus collisions therefore provides a direct probe of the antiquark densities in protons and nuclei.

The naive quark parton model predicts scaling of the Drell-Yan cross section in the variable $\tau \equiv M^{2} / S$, where $M$ is the invariant mass of the lepton pair and $\sqrt{S}$ is the hadron-hadron centre-of-mass energy. Apart from the invariant mass distribution $\mathrm{d} \sigma / \mathrm{d} M^{2}$ one usually studies the distribution of the lepton pairs as function of the Feynman-parameter

$$
x_{F} \equiv \frac{2 q_{z}}{\sqrt{S}}
$$

or of the hadron-hadron centre-of-mass rapidity

$$
y \equiv \frac{1}{2} \ln \frac{q_{0}+q_{z}}{q_{0}-q_{z}},
$$

where $q$ denotes the four-momentum of the lepton pair in the hadron-hadron centre-ofmass system. The resulting distributions

$$
\frac{\mathrm{d} \sigma}{\mathrm{d} M^{2} \mathrm{~d} x_{F}} \quad \text { and } \quad \frac{\mathrm{d} \sigma}{\mathrm{d} M^{2} \mathrm{~d} y}
$$

can be directly related to the $x$-dependence of the parton distribution functions in beam and target. Moreover, most fixed target experiments have only a limited kinematic coverage in $x_{F}$ or $y$, such that only these distributions can be measured without extrapolation into experimentally inaccessible regions.

A summary of the experimental data on the Drell-Yan process in unpolarized pp-, $p N-, \bar{p} N$ - and $\pi N$-collisions can be found in [2]. These data are used to determine the large- $x$ behaviour of antiquark distributions in the proton (e.g. [3, 国) and to study the parton structure of the pion [5]. In recent times, the difference between Drell-Yan cross sections in $p p$ and $p d$ collisions [6] has provided valuable information on the asymmetry between the $\bar{u}$ - and $\bar{d}$-quark distributions in the proton [7].

Up to now, the only experimental information on the polarized parton distributions in the proton comes from deep inelastic lepton-nucleon scattering experiments [8], measuring the polarized structure function $g_{1}\left(x, Q^{2}\right)$. This structure function probes one particular combination of the polarized quark distributions, its mere knowledge is therefore insufficient for a determination of all different quark and antiquark distributions. This lack of knowledge is reflected in the results of recent fits of polarized parton distribution functions [9, 10, 11]. While the polarized valence quark distributions can be determined with some precision from the experimental data, only little information can be extracted on the total magnitude of the polarized sea quark distribution; the flavour structure of the polarized quark sea is at present completely undetermined. 
Experimental information on the polarized antiquark distributions can be obtained from several observables. Charged current exchange in polarized electron-proton scattering can probe the polarized weak structure functions [12], which are sensitive to a different combination of quark distributions than their electromagnetic counterparts. Combining electromagnetic and weak structure functions in a global parton distribution fit, it should in principle be possible to disentangle the different quark and antiquark flavours. A direct probe of the polarized sea quark distributions would be the Drell-Yan process in polarized proton-nucleon collisions [13]. Such a measurement could be feasible at RHIC [14] or at HERA 15.

The rather large QCD corrections to the unpolarized Drell-Yan process suggest, that a reliable interpretation of the Drell-Yan cross section in terms of partonic distribution functions is only possible if QCD corrections are included. This paper aims to derive the $\mathcal{O}\left(\alpha_{s}\right)$ corrections to the $x_{F}$ and $y$-distributions of lepton pairs in longitudinally polarized hadron-hadron collisions and to study the impact of these corrections on the Drell-Yan cross section.

For the unpolarized Drell-Yan process, QCD corrections have been calculated up to $\mathcal{O}\left(\alpha_{s}^{2}\right)$ for the invariant mass distribution [16, 17] and up to $\mathcal{O}\left(\alpha_{s}\right)$ for the rapidity and $x_{F}$-distributions [18, 19]. For the latter, parts of the $\mathcal{O}\left(\alpha_{s}^{2}\right)$ corrections are known [20]. Inclusion of the $\mathcal{O}\left(\alpha_{s}\right)$-corrections changes the Drell-Yan cross section at fixed target energies by up to $50 \%$, the $\mathcal{O}\left(\alpha_{s}^{2}\right)$ corrections amount up to $10 \%$. At small transverse momenta of the lepton pair, the QCD corrections are dominated by the emission of soft gluons. The effects of soft gluon emission at arbitrary order in perturbation theory have been studied for the Drell-Yan process in [21], yielding a resummation of the dominant corrections to all orders.

All these results can in principle be applied to the production of vector bosons at hadron colliders. However, in the case of $W$-boson production, one has to take into account that $W$-bosons in hadronic collisions can be identified only in their $l \nu_{l}$ decay mode, where just the lepton is observed. All above calculations cannot make predictions for the kinematic distributions of the vector boson decay products in the hadron-hadron centre-of-mass frame. Separate calculations of the next-to-leading order QCD corrections to the transverse mass distribution of the $W$-boson [22], to the polarization density matrix of its decay products [23] and to the production rates of $W+0,1$ jets [24] in unpolarized hadronic collisions have been performed.

QCD corrections to the Drell-Yan process in polarized hadron-hadron collisions have been derived up to $\mathcal{O}\left(\alpha_{s}\right)$ for the invariant mass distribution in longitudinally [25, 26, 27] and transversely [28] polarized collisions. The $\mathcal{O}\left(\alpha_{s}\right)$ corrections to the rapidity distributions are known for longitudinally [26] and transversely [28] polarized collisions. Furthermore, a resummation of the effects of soft gluons in longitudinally polarized collisions has been performed in 26].

A fully consistent study of the Drell-Yan process at next-to-leading order was until now only possible in the unpolarized case, as the polarized parton distributions could only be determined at leading accuracy. With the recently calculated polarized two-loop splitting functions [29], the polarized distributions can now be determined to next-toleading order from fits [9, 10, 11] to polarized structure function data. Having a complete 
calculation of the two-loop splitting functions available, it is now furthermore possible to define consistent scheme transformation prescriptions [29, 11] for parton distributions, splitting functions and parton level cross sections at next-to-leading order. The scheme dependence of the parton level cross sections for polarized deep inelastic scattering has lead to some discussion [30] about the partonic interpretation of measurements [8] of polarized lepton-nucleon scattering. The scheme dependence of the QCD corrections to the polarized Drell-Yan process has been addressed in 26, 27].

This paper is organized as follows: in Section 2, we derive the $\mathcal{O}\left(\alpha_{s}\right)$-corrections to the $x_{F}$-distribution of Drell-Yan lepton pairs produced in longitudinally polarized hadronhadron collisions. We discuss the representation of $\gamma_{5}$ used in our calculation and motivate our choice of factorization scheme. Furthermore, a brief rederivation of the corrections to the invariant mass and $y$-distributions is given. We numerically estimate the importance of these corrections at fixed target energies in Section 3. Section 1 contains a brief summary of our results and concluding remarks. In the Appendix, we collect several identities used in the calculation.

\section{Perturbative corrections to the polarized Drell-Yan process}

The QCD corrections to the $x_{F}$-distributions in the unpolarized Drell-Yan process were originally derived using dimensional regularization by Altarelli, Ellis and Martinelli [18]. In a later publication, Kubar et al. [19] confirmed the results of [18] and derived the QCD corrections to the $y$-distributions. The calculation of Kubar et al. was performed using a non-zero gluon mass as regulator. The results obtained for the $x_{F}$-distributions with this method were considerably simpler than the original results of [18]. In the calculation of the QCD corrections to the $x_{F^{-}}$and $y$-distributions in the polarized Drell-Yan process, we shall work in dimensional regularization and follow closely the method of [18]. Using several identities listed in the Appendix, we are able to simplify our results into a form similar to the unpolarized results of [19]. In the following, we give an explicit derivation of the corrections to the $x_{F}$-distribution. Furthermore, we briefly summarize the results of a rederivation of QCD corrections to the $y$-distribution within the same method. These corrections were originally obtained by Weber [26] as a 'by-product' in the calculation of the soft gluon resummation in the polarized Drell-Yan process.

Disregarding correlations between the final state lepton plane and the direction of

the incoming hadrons, it is sufficient to consider the production of an off-shell photon of invariant mass $M^{2}$, the polarization sum of the outgoing photon can be taken to be $-g^{\mu \nu}$. The lepton pair production cross section can be retrieved by multiplying the results with a factor $\alpha /\left(3 \pi M^{2}\right) \mathrm{d} M^{2}$ for the decay $\gamma^{*} \rightarrow l^{+} l^{-}$.

Only the lowest order contribution to the Drell-Yan process (Fig. 11(a)) yields a finite cross section. All higher order parton level cross sections contain infinities. To make these infinities explicit, we work in dimensional regularization with $d=4-2 \epsilon$ dimensions. This treatment faces the problem of describing $\gamma_{5}$ in $n \neq 4$. We shall use the $\gamma_{5}$-prescription [31] of 't Hooft, Veltman, Breitenlohner and Maison (HVBM), which has been consistently 
used in the derivation of the next-to-leading order corrections to the polarized splitting functions [29]. In this prescription, one restricts the anticommutation property of $\gamma_{5}$ to the physical four dimensions, while $\gamma_{5}$ commutes in the remaining $n-4$ dimensions. The major drawback of this formalism in the $\overline{\mathrm{MS}}$-scheme is the non-conservation of the flavour non-singlet axial vector currents [32] due to a non-vanishing first moment of the corresponding non-singlet NLO splitting function $\Delta P_{q q,+}$. To restore the conservation of this non-singlet axial vector current, a further scheme transformation [32] of the results obtained in the HVBM formalism is needed [29]. We have to apply the same scheme transformation to our results for the parton-level Drell-Yan cross sections at $\mathcal{O}\left(\alpha_{s}\right)$.

We define the hadron cross sections:

$$
\mathrm{d} \Delta \sigma \equiv \frac{1}{2}\left(\mathrm{~d} \sigma^{++}-\mathrm{d} \sigma^{+-}\right), \quad \mathrm{d} \sigma \equiv \frac{1}{2}\left(\mathrm{~d} \sigma^{++}+\mathrm{d} \sigma^{+-}\right),
$$

where $(+)$ and $(-)$ denote positive and negative hadron helicities. In the parton model picture, these cross sections can be expressed as convolutions of (polarized) parton distributions $[\Delta] f_{i}$ with parton subprocess cross sections $[\Delta] \hat{\sigma}_{i j}$ :

$$
\mathrm{d}[\Delta] \sigma=\int \mathrm{d} x_{1} \mathrm{~d} x_{2} \sum_{i j} \mathrm{~d}[\Delta] \hat{\sigma}_{i j}\left(x_{1}, x_{2}, \mu_{F}^{2}\right)[\Delta] f_{i}\left(x_{1}, \mu_{F}^{2}\right)[\Delta] f_{j}\left(x_{2}, \mu_{F}^{2}\right) .
$$

We have defined

$$
\mathrm{d} \Delta \hat{\sigma} \equiv \frac{1}{2}\left(\mathrm{~d} \hat{\sigma}^{++}-\mathrm{d} \hat{\sigma}^{+-}\right), \quad \mathrm{d} \hat{\sigma} \equiv \frac{1}{2}\left(\mathrm{~d} \hat{\sigma}^{++}+\mathrm{d} \hat{\sigma}^{+-}\right),
$$

where $(+)$ and $(-)$ now denote positive and negative parton helicities.

The lowest order bare cross section for the $q \bar{q} \rightarrow \gamma^{*}$ contribution (Fig. 1. (a)) reads in $d=4-2 \epsilon$ dimensions:

$$
\Delta \hat{\sigma}^{0}=-\frac{4 \pi^{2} \alpha e_{q}^{2}}{3 M^{2}}(1+\epsilon) \delta(1-z),
$$

where we define $z=M^{2} / s$, with $\sqrt{s}$ being the parton-parton centre-of-mass energy. The factor $(1+\epsilon)$ is common to all contributions and forms part of the overall normalization of the cross section.

The virtual gluon correction (Fig. 1. (b)) to this process yields

$$
\Delta \hat{\sigma}^{1, V}=-\frac{4 \pi^{2} \alpha e_{q}^{2}}{3 M^{2}}(1+\epsilon) \delta(1-z) \frac{\alpha_{s}}{2 \pi} C_{F}\left(\frac{4 \pi \mu^{2}}{M^{2}}\right)^{\epsilon} \frac{1}{\Gamma(1-\epsilon)}\left(-\frac{2}{\epsilon^{2}}-\frac{3}{\epsilon}-8+\pi^{2}\right) .
$$

The correction due to real gluon emission (Fig. 1. (c,d)) takes the form:

$$
\begin{aligned}
\Delta \hat{\sigma}^{1, R} & =-\frac{4 \pi^{2} \alpha e_{q}^{2}}{3 M^{2}}(1+\epsilon) \frac{\alpha_{s}}{2 \pi} C_{F}\left(\frac{4 \pi \mu^{2}}{M^{2}}\right)^{\epsilon} \frac{1}{\Gamma(1-\epsilon)} z^{1+\epsilon}(1-z)^{1-2 \epsilon} \\
& \times \int_{0}^{1} \mathrm{~d} z_{1} z_{1}^{-\epsilon}\left(1-z_{1}\right)^{-\epsilon}\left[(1+3 \epsilon)\left(\frac{1-z_{1}}{z_{1}}+\frac{z_{1}}{1-z_{1}}\right)+\frac{2 z}{(1-z)^{2} z_{1}\left(1-z_{1}\right)}\right]
\end{aligned}
$$

\footnotetext{
${ }^{1}$ This scheme transformation was first considered by Weber [26] to restore the conservation of quark helicity in the $q \bar{q}-$ contribution to the polarized Drell-Yan process.
} 


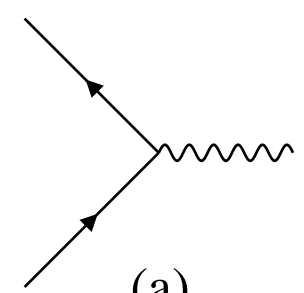

(a)

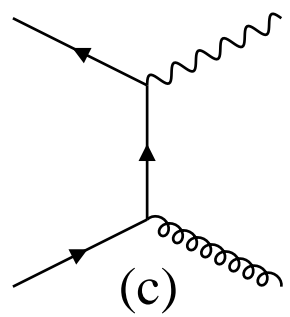

(c)

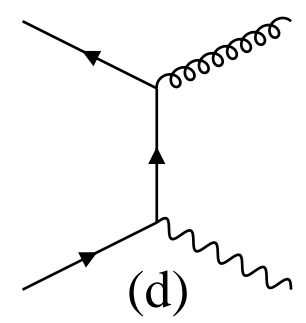

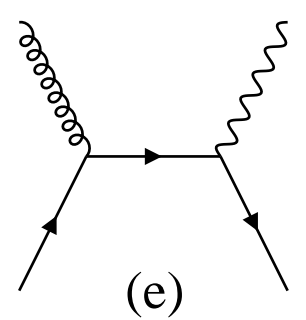

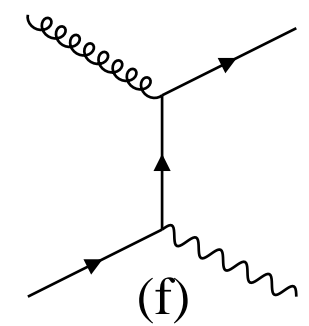

Figure 1: Parton level subprocesses contributing to the Drell-Yan process up to $\mathcal{O}\left(\alpha_{s}\right)$ : lowest order $q \bar{q}-$ annihilation (a); virtual (b) and real (c,d) gluon corrections to $q \bar{q}-$ annihilation; quark-gluon Compton scattering (e,f).

where we have replaced the parton-parton scattering angle $\Theta^{*}$ by $z_{1} \equiv\left(1+\cos \Theta^{*}\right) / 2$. Terms proportional to $\epsilon$ have been omitted if they do not yield a finite contribution to the final result. Finally, the contribution from the quark-gluon Compton scattering process (Fig. [1. (e,f)) reads:

$$
\begin{aligned}
\Delta \hat{\sigma}^{1, C} & =-\frac{4 \pi^{2} \alpha e_{q}^{2}}{3 M^{2}}(1+\epsilon) \frac{\alpha_{s}}{2 \pi} T_{F}\left(\frac{4 \pi \mu^{2}}{M^{2}}\right)^{\epsilon} \frac{1}{\Gamma(1-\epsilon)} z^{1+\epsilon}(1-z)^{1-2 \epsilon} \\
& \times \int_{0}^{1} \mathrm{~d} z_{1} z_{1}^{-\epsilon}\left(1-z_{1}\right)^{-\epsilon}\left[\frac{1}{(1-z) z_{1}}+2-(1+\epsilon) \frac{2}{z_{1}}-\left(2-z_{1}\right)(1-z)\right] .
\end{aligned}
$$

Using eqs. (A.1), one can make the poles in $\epsilon$ in the annihilation and Compton contributions explicit:

$$
\begin{aligned}
\Delta \hat{\sigma}^{1, A} \equiv & \Delta \hat{\sigma}^{1, V}+\Delta \hat{\sigma}^{1, R} \\
= & -\frac{4 \pi^{2} \alpha e_{q}^{2}}{3 M^{2}}(1+\epsilon) \frac{\alpha_{s}}{2 \pi} C_{F}\left(\frac{4 \pi \mu^{2}}{M^{2}}\right)^{\epsilon} \frac{1}{\Gamma(1-\epsilon)} \\
\times & \int_{0}^{1} \mathrm{~d} z_{1} z\left\{\left(\frac{\pi^{2}}{2}-4\right) \delta(1-z)\left(\delta\left(z_{1}\right)+\delta\left(1-z_{1}\right)\right)\right. \\
& +\delta(1-z)\left[-\frac{1}{\epsilon}\left(\frac{1}{\left(z_{1}\right)_{+}}+\frac{1}{\left(1-z_{1}\right)_{+}}\right)+\left(\frac{\ln z_{1}}{z_{1}}\right)_{+}+\left(\frac{\ln \left(1-z_{1}\right)}{1-z_{1}}\right)_{+}\right. \\
& \left.\quad+\frac{\ln z_{1}}{1-z_{1}}+\frac{\ln \left(1-z_{1}\right)}{z_{1}}\right]
\end{aligned}
$$




$$
\begin{aligned}
+ & {\left[\delta\left(z_{1}\right)+\delta\left(1-z_{1}\right)\right]\left[-\frac{1}{\epsilon}\left[\frac{2}{(1-z)_{+}}-1-z+\frac{3}{2} \delta(1-z)\right]\right.} \\
& \left.+(1-z)\left[\ln \frac{(1-z)^{2}}{z}-3\right]-2 \ln \frac{(1-z)^{2}}{z}+4\left(\frac{\ln (1-z)}{1-z}\right)_{+}-\frac{2}{1-z} \ln z\right] \\
+ & {\left.\left[\frac{1}{\left(z_{1}\right)_{+}}+\frac{1}{\left(1-z_{1}\right)_{+}}\right]\left[-1-z+\frac{2}{(1-z)_{+}}\right]-2(1-z)\right\}, } \\
\Delta \hat{\sigma}^{1, C}= & -\frac{4 \pi^{2} \alpha e_{q}^{2}}{3 M^{2}}(1+\epsilon) \frac{\alpha_{s}}{2 \pi} T_{F}\left(\frac{4 \pi \mu^{2}}{M^{2}}\right)^{\epsilon} \frac{1}{\Gamma(1-\epsilon)} \\
\times & \int_{0}^{1} \mathrm{~d} z_{1} z\left\{-\frac{1}{\epsilon} \delta\left(z_{1}\right)[2 z-1]+\delta\left(z_{1}\right)\left[(2 z-1) \ln \frac{(1-z)^{2}}{z}+2-2 z\right]\right. \\
& \left.+\frac{1}{\left(z_{1}\right)_{+}}[2 z-1]+2(1-z)-\left(2-z_{1}\right)(1-z)^{2}\right\} .
\end{aligned}
$$

The $x_{F}$-differential cross section can now be obtained by multiplying the above expressions by

$$
\int \mathrm{d} x_{F} \delta\left(x_{F}-\left[z_{1}\left(x_{1}+x_{2}\right)(1-z)+z x_{1}-x_{2}\right]\right) .
$$

Using the definitions

$$
x_{F}=x_{1}^{0}-x_{2}^{0} \quad \text { and } \quad \tau=x_{1}^{0} x_{2}^{0}
$$

one can fix

$$
x_{1}^{0}=\frac{1}{2}\left(x_{F}+\sqrt{x_{F}^{2}+4 \tau}\right), \quad x_{2}^{0}=\frac{1}{2}\left(-x_{F}+\sqrt{x_{F}^{2}+4 \tau}\right)
$$

for the $x_{F}$-distributions. The parton level cross sections $\Delta \hat{\sigma}^{1, A}$ and $\Delta \hat{\sigma}^{1, C}$ can then be rewritten using eqs. (A.3)-(A.6).

The resulting parton level cross sections still contain divergences associated with collinear singularities in the initial state. These collinear singularities are process independent and are absorbed into the bare parton distribution functions by a mass factorization procedure. The only distribution relevant in our case is the polarized quark distribution. Performing the mass factorization in the $\overline{\mathrm{MS}}$-scheme and making a scheme transformation [29, 26] to remove spurious terms due to the HVBM prescription for $\gamma_{5}$ yields for the bare quark distribution up to $\mathcal{O}\left(\alpha_{s}\right)$ :

$$
\begin{aligned}
\Delta q(x)= & \Delta q\left(x, \mu_{F}^{2}\right)-\frac{\alpha_{s}}{2 \pi} \int_{x}^{1} \frac{\mathrm{d} x^{\prime}}{x^{\prime}}\left[-\frac{1}{\epsilon} \frac{1}{\Gamma(1-\epsilon)}\left(\frac{4 \pi \mu^{2}}{\mu_{F}^{2}}\right)^{\epsilon} \Delta P_{q q}\left(x^{\prime}\right)+\Delta z_{q q}\left(x^{\prime}\right)\right] \Delta q\left(x / x^{\prime}\right) \\
& -\frac{\alpha_{s}}{2 \pi} \int_{x}^{1} \frac{\mathrm{d} x^{\prime}}{x^{\prime}}\left[-\frac{1}{\epsilon} \frac{1}{\Gamma(1-\epsilon)}\left(\frac{4 \pi \mu^{2}}{\mu_{F}^{2}}\right)^{\epsilon} \Delta P_{q g}\left(x^{\prime}\right)\right] \Delta G\left(x / x^{\prime}\right)
\end{aligned}
$$

with 33]:

$$
\begin{aligned}
& \Delta P_{q q}(x)=C_{F}\left[\frac{2}{(1-x)_{+}}-1-x+\frac{3}{2} \delta(1-x)\right], \quad \Delta z_{q q}(x)=-4 C_{F}(1-x) . \\
& \Delta P_{q g}(x)=T_{F}[2 x-1] .
\end{aligned}
$$


After mass factorization, the polarized cross sections for the Drell-Yan production of lepton pairs at NLO can be expressed in a form similar to the unpolarized cross sections [5]:

$$
\begin{aligned}
\frac{\mathrm{d} \Delta \sigma}{\mathrm{d} M^{2} \mathrm{~d} x_{F}}= & \frac{4 \pi \alpha^{2}}{9 M^{2} S} \sum_{i} e_{i}^{2} \int_{x_{1}^{0}}^{1} \mathrm{~d} x_{1} \int_{x_{2}^{0}}^{1} \mathrm{~d} x_{2} \\
\times & \left\{[ \frac { \mathrm { d } \Delta \hat { \sigma } _ { q \overline { q } } ^ { ( 0 ) } } { \mathrm { d } M ^ { 2 } \mathrm { d } x _ { F } } ( x _ { 1 } , x _ { 2 } ) + \frac { \alpha _ { s } } { 2 \pi } \frac { \mathrm { d } \Delta \hat { \sigma } _ { q \overline { q } } ^ { ( 1 ) } } { \mathrm { d } M ^ { 2 } \mathrm { d } x _ { F } } ( x _ { 1 } , x _ { 2 } , \frac { M ^ { 2 } } { \mu _ { F } ^ { 2 } } ) ] \left\{\Delta q_{i}\left(x_{1}, \mu_{F}^{2}\right) \Delta \bar{q}_{i}\left(x_{2}, \mu_{F}^{2}\right)\right.\right. \\
& \left.+\Delta \bar{q}_{i}\left(x_{1}, \mu_{F}^{2}\right) \Delta q_{i}\left(x_{2}, \mu_{F}^{2}\right)\right\} \\
+ & {\left[\frac{\alpha_{s}}{2 \pi} \frac{\mathrm{d} \Delta \hat{\sigma}_{q g}^{(1)}}{\mathrm{d} M^{2} \mathrm{~d} x_{F}}\left(x_{1}, x_{2}, \frac{M^{2}}{\mu_{F}^{2}}\right) \Delta G\left(x_{1}, \mu_{F}^{2}\right)\left\{\Delta q_{i}\left(x_{2}, \mu_{F}^{2}\right)+\Delta \bar{q}_{i}\left(x_{2}, \mu_{F}^{2}\right)\right\}\right.} \\
& +(1 \leftrightarrow 2)]\},
\end{aligned}
$$

with

$$
\begin{aligned}
& \frac{\mathrm{d} \Delta \hat{\sigma}_{q \bar{q}}^{(0)}}{\mathrm{d} M^{2} \mathrm{~d} x_{F}}\left(x_{1}, x_{2}\right)=-\frac{\delta\left(x_{1}-x_{1}^{0}\right) \delta\left(x_{2}-x_{2}^{0}\right)}{x_{1}^{0}+x_{2}^{0}}=-\frac{\mathrm{d} \hat{\sigma}_{q \bar{q}}^{(0)}}{\mathrm{d} M^{2} \mathrm{~d} x_{F}}\left(x_{1}, x_{2}\right), \\
& \frac{\mathrm{d} \Delta \hat{\sigma}_{q \bar{q}}^{(1)}}{\mathrm{d} M^{2} \mathrm{~d} x_{F}}\left(x_{1}, x_{2}, \frac{M^{2}}{\mu_{F}^{2}}\right)=-C_{F}\left\{\frac { \delta ( x _ { 1 } - x _ { 1 } ^ { 0 } ) \delta ( x _ { 2 } - x _ { 2 } ^ { 0 } ) } { x _ { 1 } ^ { 0 } + x _ { 2 } ^ { 0 } } \left[\frac{\pi^{2}}{3}-8+2 \operatorname{Li}_{2}\left(x_{1}^{0}\right)+2 \operatorname{Li}_{2}\left(x_{2}^{0}\right)\right.\right. \\
& \left.+\ln ^{2}\left(1-x_{1}^{0}\right)+\ln ^{2}\left(1-x_{2}^{0}\right)+2 \ln \frac{x_{1}^{0}}{1-x_{1}^{0}} \ln \frac{x_{2}^{0}}{1-x_{2}^{0}}\right] \\
& +\left(\frac { \delta ( x _ { 1 } - x _ { 1 } ^ { 0 } ) } { x _ { 1 } ^ { 0 } + x _ { 2 } ^ { 0 } } \left[\frac{1}{x_{2}}-\frac{x_{2}^{0}}{x_{2}^{2}}-\frac{x_{2}^{0}{ }^{2}+x_{2}^{2}}{x_{2}^{2}\left(x_{2}-x_{2}^{0}\right)} \ln \frac{x_{2}^{0}}{x_{2}}\right.\right. \\
& +\frac{x_{2}^{0}{ }^{2}+x_{2}^{2}}{x_{2}^{2}}\left(\frac{\ln \left(1-x_{2}^{0} / x_{2}\right)}{x_{2}-x_{2}^{0}}\right)_{+}+\frac{x_{2}^{0}{ }^{2}+x_{2}^{2}}{x_{2}^{2}} \frac{1}{\left(x_{2}-x_{2}^{0}\right)_{+}} \\
& \left.\left.\ln \frac{\left(x_{1}^{0}+x_{2}^{0}\right)\left(1-x_{1}^{0}\right)}{x_{1}^{0}\left(x_{1}^{0}+x_{2}\right)}\right]+(1 \leftrightarrow 2)\right) \\
& +\frac{\Delta \tilde{G}^{A}\left(x_{1}, x_{2}\right)}{\left[\left(x_{1}-x_{1}^{0}\right)\left(x_{2}-x_{2}^{0}\right)\right]_{+}}+\Delta \tilde{H}^{A}\left(x_{1}, x_{2}\right) \\
& +\ln \frac{M^{2}}{\mu_{F}^{2}}\left\{\frac{\delta\left(x_{1}-x_{1}^{0}\right) \delta\left(x_{2}-x_{2}^{0}\right)}{x_{1}^{0}+x_{2}^{0}}\left[3+2 \ln \frac{1-x_{1}^{0}}{x_{1}^{0}}+2 \ln \frac{1-x_{2}^{0}}{x_{2}^{0}}\right]\right. \\
& \left.\left.+\left(\frac{\delta\left(x_{1}-x_{1}^{0}\right)}{x_{1}^{0}+x_{2}^{0}} \frac{x_{2}^{0}{ }^{2}+x_{2}^{2}}{x_{2}^{2}} \frac{1}{\left(x_{2}-x_{2}^{0}\right)_{+}}+(1 \leftrightarrow 2)\right)\right\}\right\} \\
& =-\frac{\mathrm{d} \hat{\sigma}_{q \bar{q}}^{(1)}}{\mathrm{d} M^{2} \mathrm{~d} x_{F}}\left(x_{1}, x_{2}, \frac{M^{2}}{\mu_{F}^{2}}\right) \text {, } \\
& \frac{\mathrm{d} \Delta \hat{\sigma}_{q g}^{(1)}}{\mathrm{d} M^{2} \mathrm{~d} x_{F}}\left(x_{1}, x_{2}, \frac{M^{2}}{\mu_{F}^{2}}\right)=-T_{F}\left\{\frac { \delta ( x _ { 2 } - x _ { 2 } ^ { 0 } ) } { ( x _ { 1 } ^ { 0 } + x _ { 2 } ^ { 0 } ) x _ { 1 } ^ { 2 } } \left[\left(2 x_{1}^{0}-x_{1}\right) \ln \frac{\left(x_{1}^{0}+x_{2}^{0}\right)\left(1-x_{2}^{0}\right)\left(x_{1}-x_{1}^{0}\right)}{x_{1}^{0} x_{2}^{0}\left(x_{1}+x_{2}^{0}\right)}\right.\right. \\
& \left.+2\left(x_{1}-x_{1}^{0}\right)\right]+\frac{\Delta \tilde{G}^{C}\left(x_{1}, x_{2}\right)}{\left(x_{2}-x_{2}^{0}\right)_{+}}+\Delta \tilde{H}^{C}\left(x_{1}, x_{2}\right)
\end{aligned}
$$




$$
\left.+\ln \frac{M^{2}}{\mu_{F}^{2}}\left\{\frac{\delta\left(x_{2}-x_{2}^{0}\right)}{\left(x_{1}^{0}+x_{2}^{0}\right) x_{1}^{2}}\left(2 x_{1}^{0}-x_{1}\right)\right\}\right\}
$$

where

$$
\begin{aligned}
\Delta \tilde{G}^{A}\left(x_{1}, x_{2}\right) & =\frac{\left(x_{1}+x_{2}\right)\left(x_{1}^{0} x_{2}^{0} 2+x_{1}^{2} x_{2}^{2}\right)}{x_{1}^{2} x_{2}^{2}\left(x_{1}^{0}+x_{2}\right)\left(x_{1}+x_{2}^{0}\right)}, \\
\Delta \tilde{H}^{A}\left(x_{1}, x_{2}\right) & =-\frac{2}{x_{1} x_{2}\left(x_{1}+x_{2}\right)}, \\
\Delta \tilde{G}^{C}\left(x_{1}, x_{2}\right) & =\frac{2 x_{1}^{0} x_{2}^{0}-x_{1} x_{2}}{x_{1}^{2} x_{2}\left(x_{1}^{0}+x_{2}\right)}, \\
\Delta \tilde{H}^{C}\left(x_{1}, x_{2}\right) & =\frac{x_{1}\left(x_{1}^{0}+x_{2}\right)\left(x_{2}-x_{2}^{0}\right)+2 x_{1}^{0} x_{2}^{0}\left(x_{1}+x_{2}\right)}{x_{1}^{2} x_{2}^{2}\left(x_{1}+x_{2}\right)^{2}} .
\end{aligned}
$$

Inserting

$$
\int \mathrm{d} y \delta\left(y-\frac{1}{2}\left[\ln \frac{x_{1}}{x_{2}}+\ln \frac{z+z_{1}-z z_{1}}{1-z_{1}+z z_{1}}\right]\right)
$$

into eqs.(10)-(11) and using identities (A.5), (A.7)-(A.9), one can derive the $\mathcal{O}\left(\alpha_{s}\right)-$ corrections to the rapidity distribution of the Drell-Yan pairs. The variables

$$
x_{1}^{0}=\sqrt{\tau} e^{y}, \quad x_{2}^{0}=\sqrt{\tau} e^{-y}
$$

are in this case defined by

$$
y=\frac{1}{2} \ln \frac{x_{1}^{0}}{x_{2}^{0}} \quad \text { and } \quad \tau=x_{1}^{0} x_{2}^{0} .
$$

They are in general different from the $x_{1}^{0}$ and $x_{2}^{0}$ occuring in the $x_{F}$-distributions.

These corrections have already been calculated in [26]; we find numerical agreement with the results (keeping in mind that a different factorization prescription for the gluonic contribution was used in [26]), our expressions are however considerably simpler:

$$
\begin{aligned}
\frac{\mathrm{d} \Delta \sigma}{\mathrm{d} M^{2} \mathrm{~d} y}= & \frac{4 \pi \alpha^{2}}{9 M^{2} S} \sum_{i} e_{i}^{2} \int_{x_{1}^{0}}^{1} \mathrm{~d} x_{1} \int_{x_{2}^{0}}^{1} \mathrm{~d} x_{2} \\
\times & \left\{[ \frac { \mathrm { d } \Delta \hat { \sigma } _ { q \overline { q } } ^ { ( 0 ) } } { \mathrm { d } M ^ { 2 } \mathrm { d } y } ( x _ { 1 } , x _ { 2 } ) + \frac { \alpha _ { s } } { 2 \pi } \frac { \mathrm { d } \Delta \hat { \sigma } _ { q \overline { q } } ^ { ( 1 ) } } { \mathrm { d } M ^ { 2 } \mathrm { d } y } ( x _ { 1 } , x _ { 2 } , \frac { M ^ { 2 } } { \mu _ { F } ^ { 2 } } ) ] \left\{\Delta q_{i}\left(x_{1}, \mu_{F}^{2}\right) \Delta \bar{q}_{i}\left(x_{2}, \mu_{F}^{2}\right)\right.\right. \\
& \left.+\Delta \bar{q}_{i}\left(x_{1}, \mu_{F}^{2}\right) \Delta q_{i}\left(x_{2}, \mu_{F}^{2}\right)\right\} \\
+ & {\left[\frac{\alpha_{s}}{2 \pi} \frac{\mathrm{d} \Delta \hat{\sigma}_{q g}^{(1)}}{\mathrm{d} M^{2} \mathrm{~d} y}\left(x_{1}, x_{2}, \frac{M^{2}}{\mu_{F}^{2}}\right) \Delta G\left(x_{1}, \mu_{F}^{2}\right)\left\{\Delta q_{i}\left(x_{2}, \mu_{F}^{2}\right)+\Delta \bar{q}_{i}\left(x_{2}, \mu_{F}^{2}\right)\right\}\right.} \\
& +(1 \leftrightarrow 2)]\}
\end{aligned}
$$


with

$$
\begin{aligned}
& \frac{\mathrm{d} \Delta \hat{\sigma}_{q \bar{q}}^{(0)}}{\mathrm{d} M^{2} \mathrm{~d} y}\left(x_{1}, x_{2}\right)=-\delta\left(x_{1}-x_{1}^{0}\right) \delta\left(x_{2}-x_{2}^{0}\right)=-\frac{\mathrm{d} \hat{\sigma}_{q \bar{q}}^{(0)}}{\mathrm{d} M^{2} \mathrm{~d} y}\left(x_{1}, x_{2}\right), \\
& \frac{\mathrm{d} \Delta \hat{\sigma}_{q \bar{q}}^{(1)}}{\mathrm{d} M^{2} \mathrm{~d} y}\left(x_{1}, x_{2}, \frac{M^{2}}{\mu_{F}^{2}}\right)=-C_{F}\left\{\delta ( x _ { 1 } - x _ { 1 } ^ { 0 } ) \delta ( x _ { 2 } - x _ { 2 } ^ { 0 } ) \left[\frac{\pi^{2}}{3}-8+2 \operatorname{Li}_{2}\left(x_{1}^{0}\right)+2 \operatorname{Li}_{2}\left(x_{2}^{0}\right)\right.\right. \\
& \left.+\ln ^{2}\left(1-x_{1}^{0}\right)+\ln ^{2}\left(1-x_{2}^{0}\right)+2 \ln \frac{x_{1}^{0}}{1-x_{1}^{0}} \ln \frac{x_{2}^{0}}{1-x_{2}^{0}}\right] \\
& +\left(\delta ( x _ { 1 } - x _ { 1 } ^ { 0 } ) \left[\frac{1}{x_{2}}-\frac{x_{2}^{0}}{x_{2}^{2}}-\frac{x_{2}^{0}{ }^{2}+x_{2}^{2}}{x_{2}^{2}\left(x_{2}-x_{2}^{0}\right)} \ln \frac{x_{2}^{0}}{x_{2}}\right.\right. \\
& +\frac{x_{2}^{02}+x_{2}^{2}}{x_{2}^{2}}\left(\frac{\ln \left(1-x_{2}^{0} / x_{2}\right)}{x_{2}-x_{2}^{0}}\right)_{+}+\frac{x_{2}^{02}+x_{2}^{2}}{x_{2}^{2}} \frac{1}{\left(x_{2}-x_{2}^{0}\right)_{+}} \\
& \left.\left.\ln \frac{2 x_{2}^{0}\left(1-x_{1}^{0}\right)}{x_{1}^{0}\left(x_{2}+x_{2}^{0}\right)}\right]+(1 \leftrightarrow 2)\right) \\
& +\frac{\Delta G^{A}\left(x_{1}, x_{2}\right)}{\left[\left(x_{1}-x_{1}^{0}\right)\left(x_{2}-x_{2}^{0}\right)\right]_{+}}+\Delta H^{A}\left(x_{1}, x_{2}\right) \\
& +\ln \frac{M^{2}}{\mu_{F}^{2}}\left\{\delta\left(x_{1}-x_{1}^{0}\right) \delta\left(x_{2}-x_{2}^{0}\right)\left[3+2 \ln \frac{1-x_{1}^{0}}{x_{1}^{0}}+2 \ln \frac{1-x_{2}^{0}}{x_{2}^{0}}\right]\right. \\
& \left.\left.+\left(\delta\left(x_{1}-x_{1}^{0}\right) \frac{x_{2}^{0}{ }^{2}+x_{2}^{2}}{x_{2}^{2}} \frac{1}{\left(x_{2}-x_{2}^{0}\right)_{+}}+(1 \leftrightarrow 2)\right)\right\}\right\} \\
& =-\frac{\mathrm{d} \hat{\sigma}_{q \bar{q}}^{(1)}}{\mathrm{d} M^{2} \mathrm{~d} y}\left(x_{1}, x_{2}, \frac{M^{2}}{\mu_{F}^{2}}\right), \\
& \frac{\mathrm{d} \Delta \hat{\sigma}_{q g}^{(1)}}{\mathrm{d} M^{2} \mathrm{~d} y}\left(x_{1}, x_{2}, \frac{M^{2}}{\mu_{F}^{2}}\right)=-T_{F}\left\{\frac { \delta ( x _ { 2 } - x _ { 2 } ^ { 0 } ) } { x _ { 1 } ^ { 2 } } \left[\left(2 x_{1}^{0}-x_{1}\right) \ln \frac{2\left(x_{1}-x_{1}^{0}\right)\left(1-x_{2}^{0}\right)}{\left(x_{1}+x_{1}^{0}\right) x_{2}^{0}}\right.\right. \\
& \left.+2\left(x_{1}-x_{1}^{0}\right)\right]+\frac{\Delta G^{C}\left(x_{1}, x_{2}\right)}{\left(x_{2}-x_{2}^{0}\right)_{+}}+\Delta H^{C}\left(x_{1}, x_{2}\right) \\
& \left.+\ln \frac{M^{2}}{\mu_{F}^{2}}\left\{\frac{\delta\left(x_{2}-x_{2}^{0}\right)}{x_{1}^{2}}\left(2 x_{1}^{0}-x_{1}\right)\right\}\right\} \text {, }
\end{aligned}
$$

where

$$
\begin{aligned}
\Delta G^{A}\left(x_{1}, x_{2}\right) & =\frac{2\left(x_{1} x_{2}+x_{1}^{0} x_{2}^{0}\right)\left(x_{1}^{0}{ }^{2} x_{2}^{0}{ }^{2}+x_{1}^{2} x_{2}^{2}\right)}{x_{1}^{2} x_{2}^{2}\left(x_{1}+x_{1}^{0}\right)\left(x_{2}+x_{2}^{0}\right)}, \\
\Delta H^{A}\left(x_{1}, x_{2}\right) & =-\frac{4 x_{1}^{0} x_{2}^{0}\left(x_{1}^{0} x_{2}^{0}+x_{1} x_{2}\right)}{x_{1} x_{2}\left(x_{1} x_{2}^{0}+x_{2} x_{1}^{0}\right)^{2}}, \\
\Delta G^{C}\left(x_{1}, x_{2}\right) & =\frac{2 x_{2}^{0}\left(2 x_{1}^{0} x_{2}^{0}-x_{1} x_{2}\right)\left(x_{1}^{0} x_{2}^{0}+x_{1} x_{2}\right)}{x_{1}^{2} x_{2}\left(x_{1} x_{2}^{0}+x_{2} x_{1}^{0}\right)\left(x_{2}+x_{2}^{0}\right)}, \\
\Delta H^{C}\left(x_{1}, x_{2}\right) & =\frac{2 x_{1}^{0} x_{2}^{0}\left(x_{1}^{0} x_{2}^{0}+x_{1} x_{2}\right)\left(x_{1} x_{1}^{0} x_{2}^{2}+x_{1}^{0} x_{2}^{0}\left(x_{1} x_{2}^{0}+2 x_{1}^{0} x_{2}\right)\right)}{x_{1}^{2} x_{2}^{2}\left(x_{1} x_{2}^{0}+x_{2} x_{1}^{0}\right)^{3}} .
\end{aligned}
$$


As mentioned above, the $\mathcal{O}\left(\alpha_{s}\right)$-corrections to the $x_{F^{-}}$and $y$-distributions in the unpolarized Drell-Yan process were derived in [18, 19]. A summary of these formulae in the $\overline{\mathrm{MS}}$-scheme in our notation can be found in [5]

Finally, integration of eqs.(10)-(11) with respect to $z_{1}$ reproduces after mass factorization using eq. (13) the correction terms to the Drell-Yan mass distribution [25, 26, 27]. These read in the $\overline{\mathrm{MS}}$-scheme:

$$
\begin{aligned}
& \frac{\mathrm{d} \Delta \sigma}{\mathrm{d} M^{2}}=\frac{4 \pi \alpha^{2}}{9 S M^{2}} \int_{0}^{1} \mathrm{~d} x_{1} \mathrm{~d} x_{2} \mathrm{~d} z \delta\left(x_{1} x_{2} z-\tau\right) \sum_{q} e_{q}^{2} \\
& \quad \times\left\{\left\{\Delta q\left(x_{1}, \mu_{F}^{2}\right) \Delta \bar{q}\left(x_{2}, \mu_{F}^{2}\right)+(1 \leftrightarrow 2)\right\}\left(\Delta c_{q \bar{q}}^{D Y,(0)}(z)+\frac{\alpha_{s}}{2 \pi} \Delta c_{q \bar{q}}^{D Y,(1)}\left(z, \frac{M^{2}}{\mu_{F}^{2}}\right)\right)\right. \\
& \left.+\left\{\left(\Delta q\left(x_{1}, \mu_{F}^{2}\right)+\Delta \bar{q}\left(x_{1}, \mu_{F}^{2}\right)\right) \Delta G\left(x_{2}, \mu_{F}^{2}\right)+(1 \leftrightarrow 2)\right\} \frac{\alpha_{s}}{2 \pi} \Delta c_{q g}^{D Y,(1)}\left(z, \frac{M^{2}}{\mu_{F}^{2}}\right)\right\},
\end{aligned}
$$

with:

$$
\begin{aligned}
\Delta c_{q \bar{q}}^{D Y,(0)}(z)= & -\delta(1-z)=-c_{q \bar{q}}^{D Y,(0)}(z) \\
\Delta c_{q \bar{q}}^{D Y,(1)}\left(z, \frac{M^{2}}{\mu_{F}^{2}}\right)= & -C_{F}\left[8\left(\frac{\ln (1-z)}{1-z}\right)_{+}-2 \frac{1+z^{2}}{1-z} \ln z-4(1+z) \ln (1-z)\right. \\
& +\delta(1-z)\left(-8+\frac{2 \pi^{2}}{3}\right) \\
& \left.+2 \ln \frac{M^{2}}{\mu_{F}^{2}}\left\{\left(\frac{2}{1-z}\right)_{+}-1-z+\frac{3}{2} \delta(1-z)\right\}\right], \\
= & -c_{q \bar{q}}^{D Y,(1)}\left(z, \frac{M^{2}}{\mu_{F}^{2}}\right) \\
\Delta c_{q g}^{D Y,(1)}\left(z, \frac{M^{2}}{\mu_{F}^{2}}\right)= & -T_{F}\left[(2 z-1) \ln \frac{(1-z)^{2}}{z}+\frac{5}{2}-z-\frac{3}{2} z^{2}+\ln \frac{M^{2}}{\mu_{F}^{2}}\{2 z-1\}\right] .
\end{aligned}
$$

\section{Numerical results}

In the following, we will illustrate the impact of the QCD corrections to the polarized Drell-Yan process derived above by numerically evaluating (14) and (19) for recent parameterizations of the polarized parton distribution functions.

When using the polarized GS parton distribution functions [9], we take $\Lambda_{n_{f}=4}^{\mathrm{QCD}}=$ $231 \mathrm{MeV}$, the corresponding unpolarized cross sections are then evaluated using the unpolarized MRS parton distribution functions set $\mathrm{A}^{\prime}$ [3]. The polarized GRSV distributions [10] are consistently used in combination with the unpolarized distributions from GRV [4] and for $\Lambda_{n_{f}=4}^{\mathrm{QCD}}=200 \mathrm{MeV}$. If not stated otherwise, we shall always use $\mu_{F}=M$; the strong coupling constant $\alpha_{s}$ is evaluated at $\mu_{F}$.

\footnotetext{
${ }^{2}$ As the normalization of the gluon polarization sum used in [5, 18, 19 deviates from the normalization conventionally used in dimensional regularization, eqs.(A8) and (A20) of [5] have to be modified according to [34] to conform to the normalization used in the NLO splitting functions and parton distributions.
} 


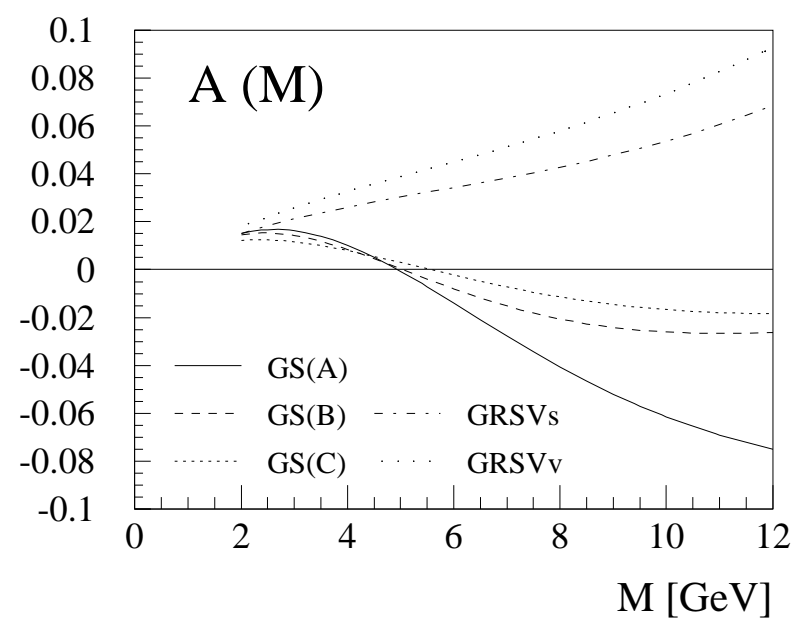

Figure 2: The Drell-Yan asymmetry $A(M)$ in $p p$ collisions with $E_{\text {beam }}=820 \mathrm{GeV}$ for different parameterizations of the polarized parton distribution functions.

All results in this section are obtained for $\sqrt{s}=39.22 \mathrm{GeV}$, corresponding to a fixed target experiment in the HERA proton beam [15] with $E_{\text {beam }}=820 \mathrm{GeV}$.

At present, all polarized parton distributions are fitted to data on the polarized structure function $g_{1}\left(x, Q^{2}\right)$ only. As this structure function is dominated by valence quark contributions for $x>0.1$, the knowledge on the polarized sea quark distributions in the large- $x$ region is very poor. This uncertainty is illustrated in Fig. 2, which shows the next-to-leading order predictions (Eq. (23)) for the Drell-Yan asymmetry

$$
A(M) \equiv \frac{\mathrm{d} \Delta \sigma}{\mathrm{d} M} / \frac{\mathrm{d} \sigma}{\mathrm{d} M}
$$

obtained using the polarized parton distribution functions of [9] $(\mathrm{GS}(\mathrm{A}-\mathrm{C}))$ and [10] (GRSVs,v). At present, neither magnitude nor sign of this asymmetry can be predicted. It should therefore be clear, that all numerical results for the polarized Drell-Yan process presented in the remainder of this section do not make solid predictions for observable asymmetries. The purpose of these numerical calculations is only to illustrate the magnitude of the next-to-leading order corrections and to demonstrate the perturbative stability of the predictions.

In the following, we shall always study the cross section asymmetries

$$
A\left(x_{F}\right) \equiv \frac{\mathrm{d} \Delta \sigma}{\mathrm{d} M \mathrm{~d} x_{F}} / \frac{\mathrm{d} \sigma}{\mathrm{d} M \mathrm{~d} x_{F}} \quad \text { and } \quad A(y) \equiv \frac{\mathrm{d} \Delta \sigma}{\mathrm{d} M \mathrm{~d} y} / \frac{\mathrm{d} \sigma}{\mathrm{d} M \mathrm{~d} y} .
$$

As the unpolarized parton distributions are known to some level of accuracy, it is possible to predict the cross sections in the denominator of $A\left(x_{F}\right)$ and $A(y)$. These cross sections are shown for proton-proton collisions $(\sqrt{s}=39.22 \mathrm{GeV})$ in Fig. 3, which is obtained with the $\operatorname{MRS}\left(\mathrm{A}^{\prime}\right)$ parton distributions [3]. The prediction using the GRV parton distributions 国 agrees within $3 \%$ over the whole kinematic range. 

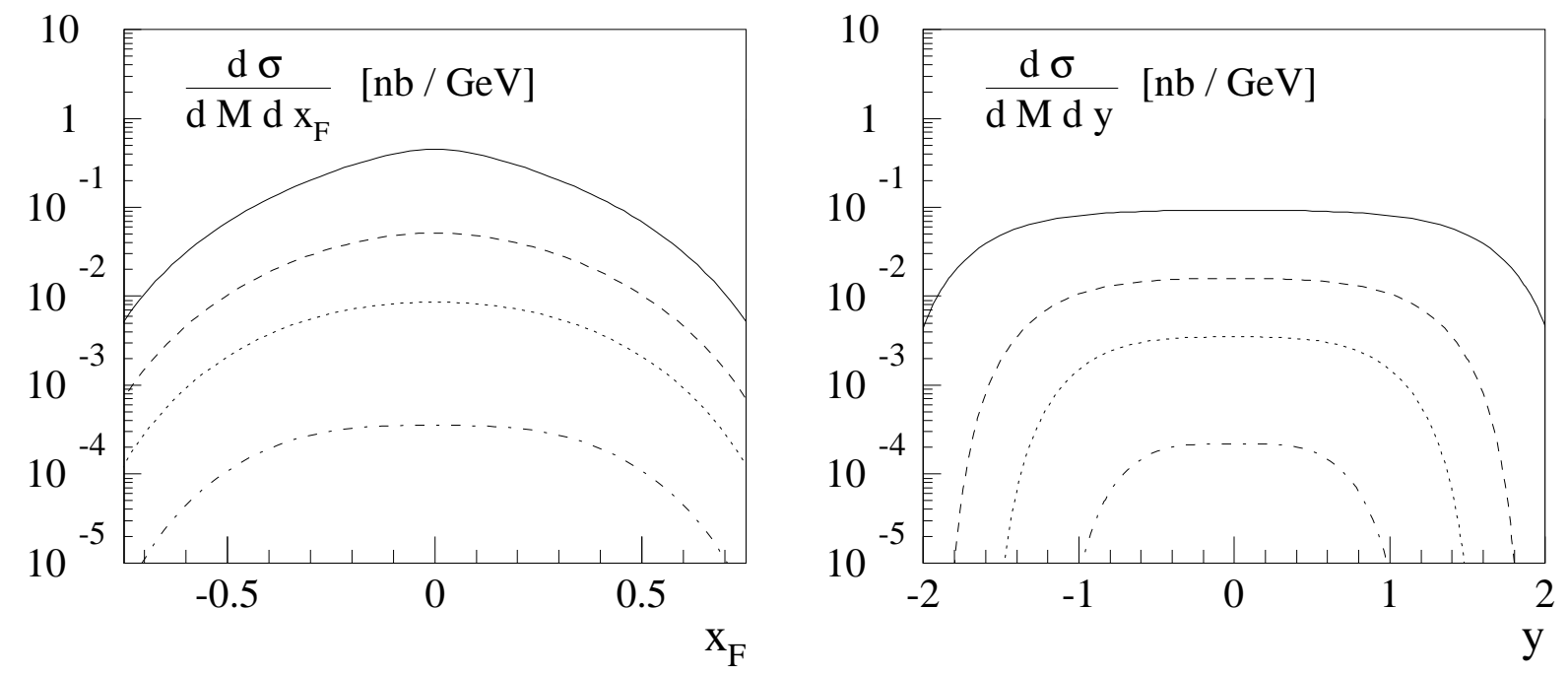

Figure 3: The unpolarized Drell-Yan cross section in $p p$ collisions. Curves are for different invariant masses $M$; solid: $4 \mathrm{GeV}$, dashed: $6 \mathrm{GeV}$, dotted: $8 \mathrm{GeV}$, dot-dashed: $12 \mathrm{GeV}$.
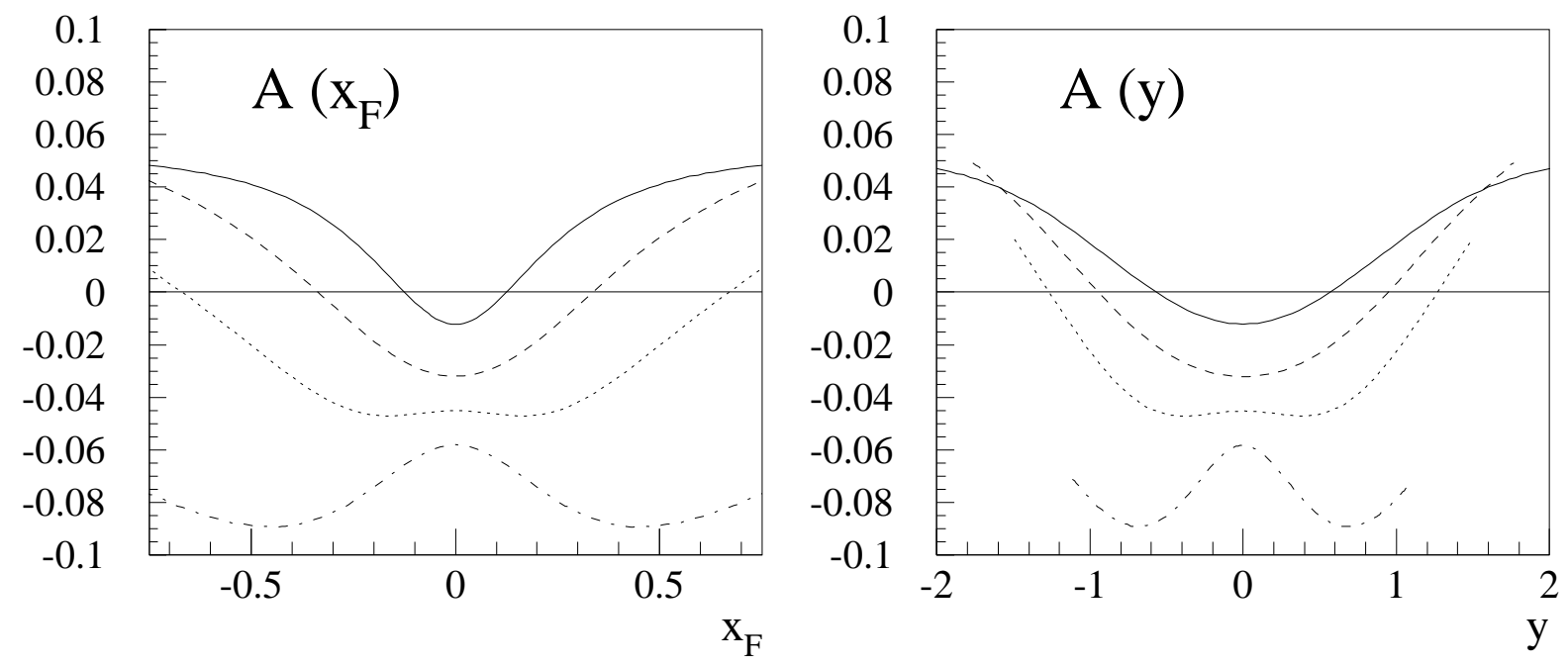

Figure 4: The Drell-Yan asymmetry in $p p$ collisions for the polarized GS(A) parton distributions. Curves are for different invariant masses $M$; solid: $4 \mathrm{GeV}$, dashed: $6 \mathrm{GeV}$, dotted: $8 \mathrm{GeV}$, dot-dashed: $12 \mathrm{GeV}$.

It can be seen from Fig. 2 that the total Drell-Yan asymmetry $A(M)$ changes with the invariant mass $M$. The change is however not uniform over the whole range in $x_{F}$ or $y$ as illustrated in Fig. 4 for the polarized GS(A) parton distributions. The $x_{F^{-}}$ dependence of the asymmetry can be understood from eq. (12). The asymmetry around $x_{F}=0$ always probes the polarized parton distributions in beam (1) and target (2) around $x_{1}^{0} \approx x_{2}^{0} \approx \sqrt{\tau}$. If $x_{F}$ is increased towards its positive kinematic limit, $x_{1}^{0}$ increases towards 

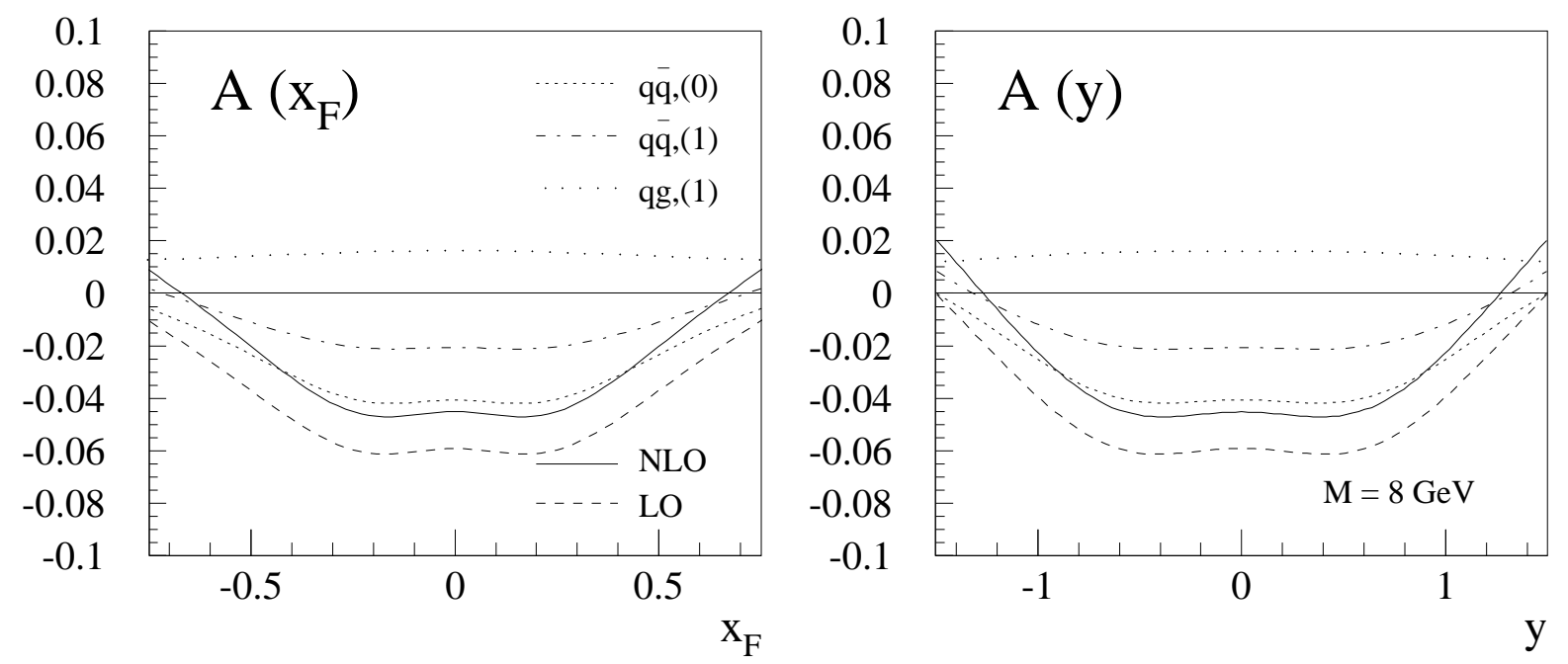

Figure 5: Contributions of the individual parton level subprocesses to the polarized DrellYan cross section. The subprocess cross sections (short-dashed, dot-dashed and dotted lines) and their sum (solid line) are normalized to the full unpolarized cross section at NLO. The long-dashed line shows the asymmetry evaluated at leading order.
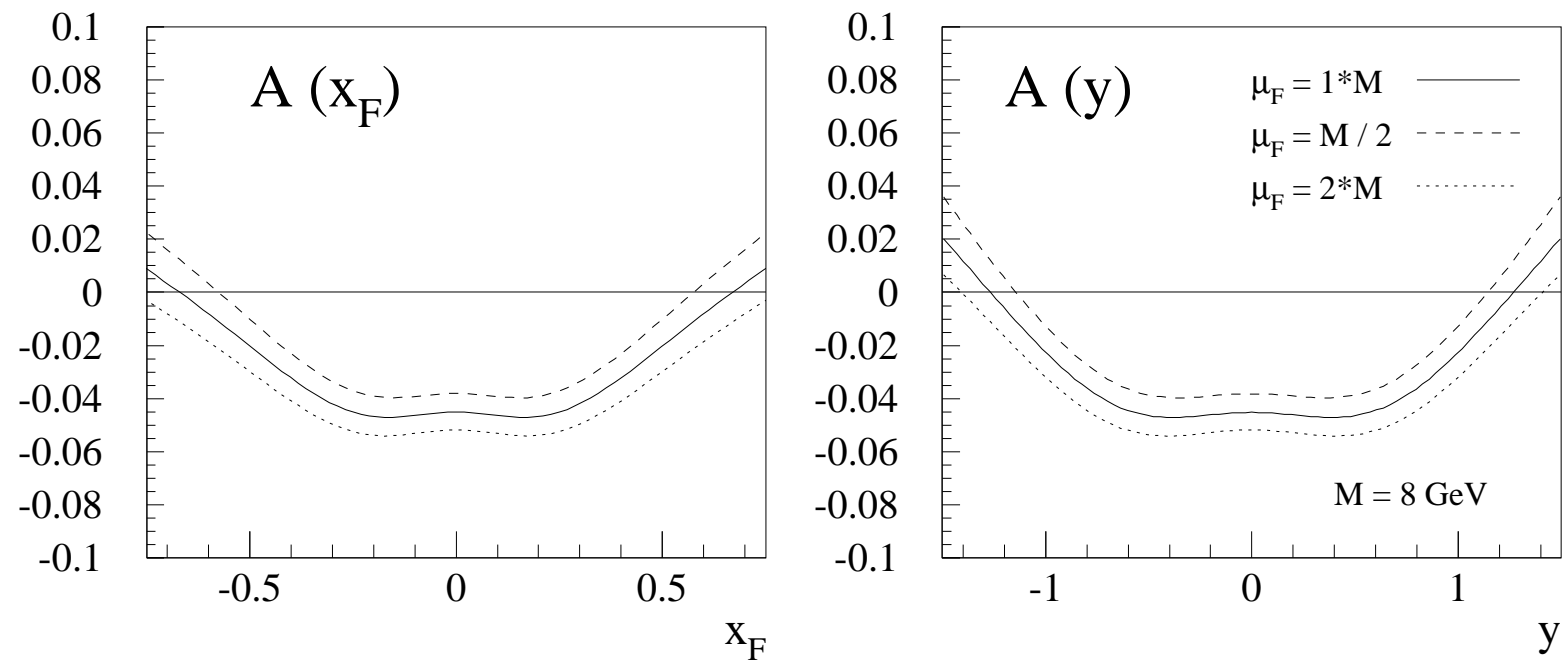

Figure 6: Sensitivity of the Drell-Yan asymmetry on variations of the factorization scale.

1 while $x_{2}^{0}$ decreases only slightly. Decreasing $x_{F}$ towards the negative kinematic limit yields a small decrease of $x_{1}^{0}$ while $x_{2}^{0}$ increases towards 1 . The region $x_{F}>0$ therefore probes the $x$-dependence of the parton distributions in the beam hadron, while $x_{F}<0$ is sensitive on the target hadron structure. Moreover, different invariant masses correspond to different $x$ intervals probed. A similar argumentation, based on eq. (18), applies to the $y$-dependence of the asymmetry.

The contributions of the individual subprocesses $(q \bar{q}-$ annihilation at leading and next- 
to-leading order and quark-gluon Compton scattering) to the polarized Drell-Yan cross section are illustrated in Fig. 5. All curves are obtained with the polarized GS(A) parton densities and are shown for $M=8 \mathrm{GeV}$. All polarized subprocess contributions are normalized to the full unpolarized cross section at next-to-leading order. The relative magnitude of the individual contributions is similar to the unpolarized Drell-Yan process. The $\mathcal{O}\left(\alpha_{s}\right)$ correction to the $q \bar{q}$-annihilation process enhances significantly the lowest order prediction while the quark-gluon Compton process contributes with a sign opposite to the annihilation process. However, the relative magnitude of annihilation and Compton process depends on the magnitude of the gluon distribution at large $x$, which is completely undetermined at present [9, 10]. This uncertainty prevents a sensible prediction of a $K-$ factor between the cross sections at leading and next-to-leading order.

Furthermore, Fig. 5 shows the prediction for the Drell-Yan asymmetry if both polarized and unpolarized cross section are evaluated using the leading order $q \bar{q},(0)$-coefficient function only (long-dashed line). The sizable shift between the leading and next-toleading order predictions for the Drell-Yan asymmetry seems to contradict the claim [25] that the next-to-leading order corrections, although large in polarized and unpolarized cross sections, would largely cancel in the Drell-Yan asymmetry.

The change of physical quantities such as cross sections or asymmetries under variations of the (unphysical) mass factorization scale $\mu_{F}$ enables an estimate of the numerical importance of unknown higher order corrections. We quantify this uncertainty in Fig. 6, showing the Drell-Yan asymmetry for $\mu_{F}=0.5,1,2 M$. It can be seen that the absolute value of the asymmetry changes less than 0.01 in the central region. Even in the region where $x_{F}$ (or $y$ ) approaches its kinematic limit, the absolute value of the asymmetry changes only by a maximum of 0.015 . This stability is crucial for an estimate of the theoretical error on the polarized sea quark distributions extracted from the polarized Drell-Yan process.

A final point briefly concerns phenomenological aspects of the polarized Drell-Yan process, although this is not the principal aim of the present paper. Figures 7 and 8 show the Drell-Yan asymmetry in polarized proton-proton and proton-deuterium collisions for $M=8 \mathrm{GeV}$ as predicted by using the different polarized parton distributions suggested in [9] and [10]. It can clearly be seen that the different predictions vary within a band of \pm 0.06 in $p p$ and \pm 0.04 in $p d$ collisions. This uncertainty reflects the present lack of knowledge on the polarization of the light quark sea in the proton at $x \gtrsim 0.1$. The difference between the predictions is furthermore substantially larger than the theoretical uncertainty due to the choice of the factorization scale.

Due to the smaller overall valence quark polarization in deuterium, one finds the asymmetry in $p d$ collisions to be systematically smaller than in $p p$ collisions. Although the normalization of the asymmetry in the proton direction $\left(x_{F}, y>0\right)$ and in the deuteron direction $\left(x_{F}, y<0\right)$ is slightly different, its shape is still very similar in both regions. This reflects the assumption of an $\mathrm{SU}(2)_{f}$-symmetry of the polarized antiquark sea, which is used in all polarized parton distributions displayed in the figure. A measurement of the $x_{F^{-}}$(or $y$-)dependence of the Drell-Yan asymmetry in $p d$ collisions could therefore test this assumption. 

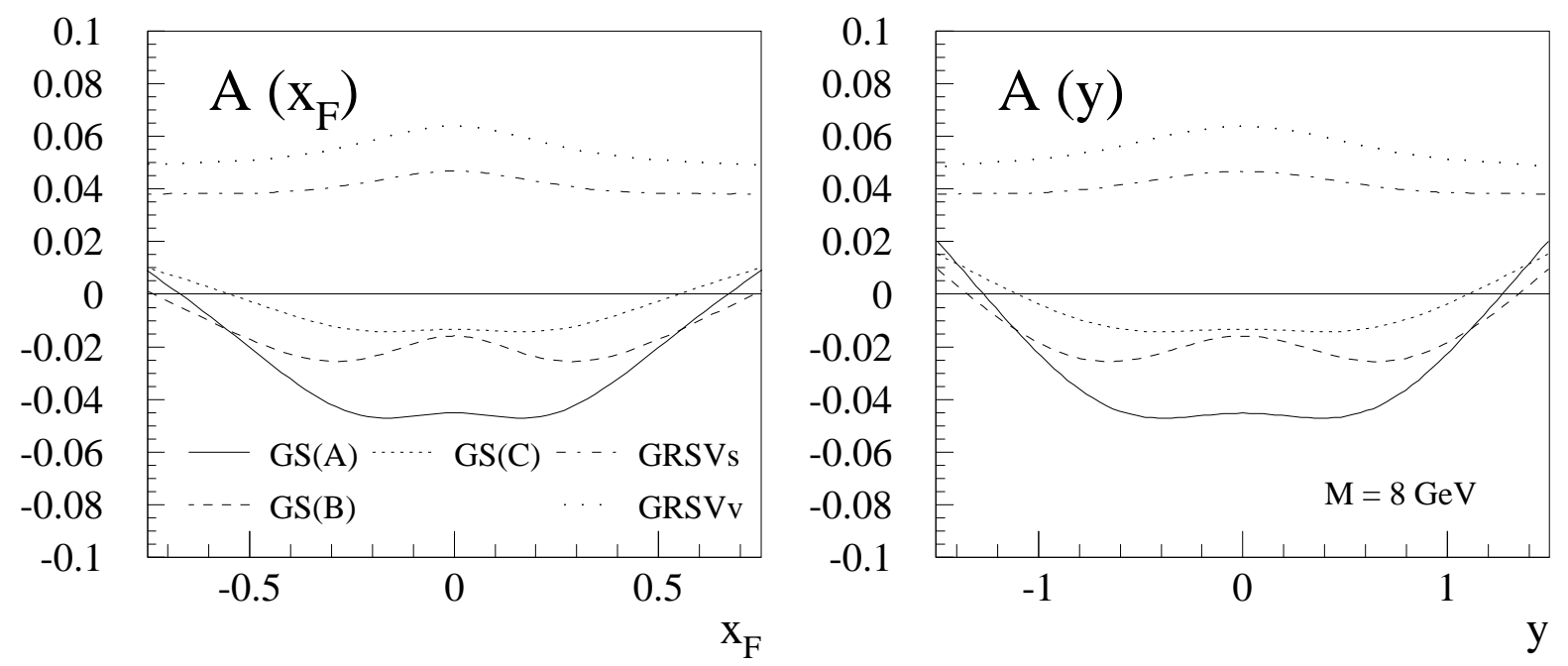

Figure 7: Predictions for the Drell-Yan asymmetry at $M=8 \mathrm{GeV}$ in polarized $p p$ collisions $\left(E_{\text {beam }}=820 \mathrm{GeV}\right)$ obtained for different parameterizations of the polarized parton densities.
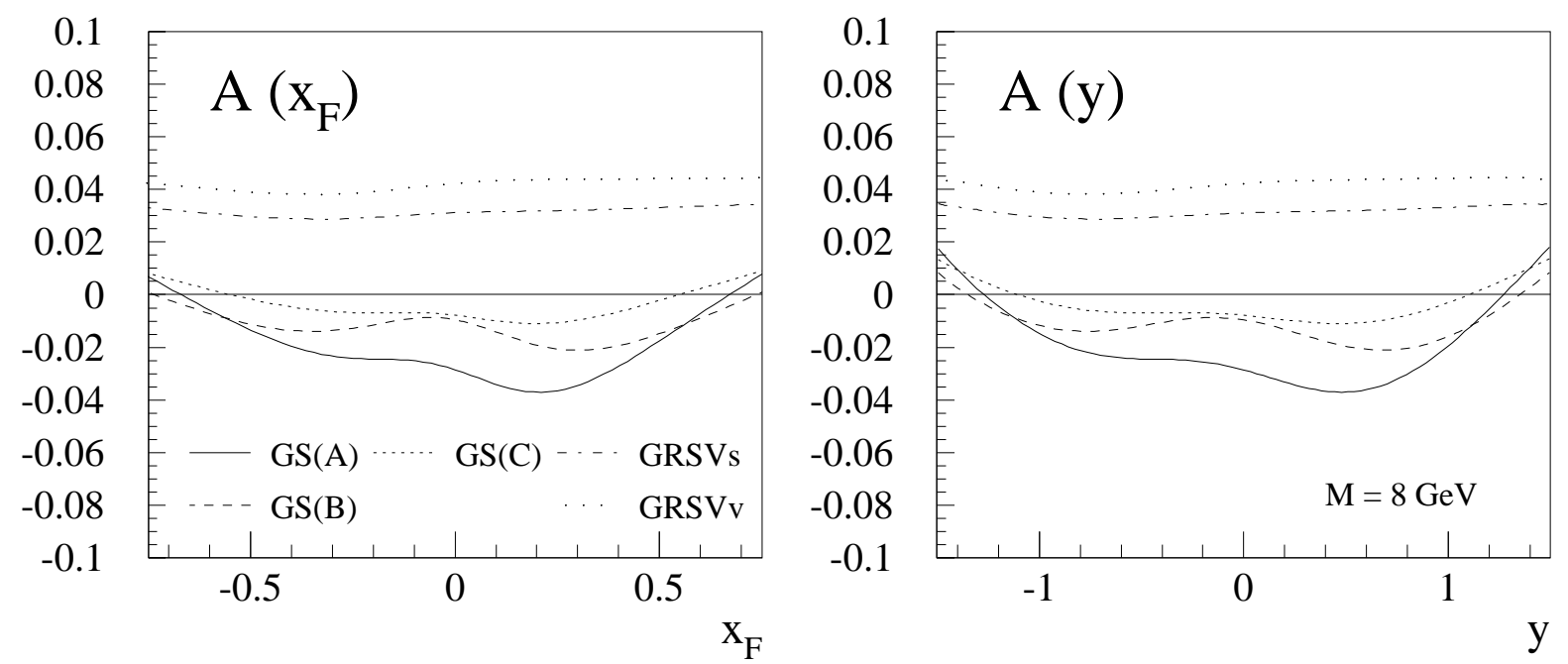

Figure 8: Predictions for the Drell-Yan asymmetry at $M=8 \mathrm{GeV}$ in polarized $p d$ collisions $\left(E_{\text {beam }}=820 \mathrm{GeV}\right)$ obtained for different parameterizations of the polarized parton densities. The proton is taken in $x_{1}$-direction.

\section{Conclusions}

We have presented a complete calculation of the $\mathcal{O}\left(\alpha_{s}\right)$ corrections to the $x_{F^{-}}$and $y$ dependence of the longitudinally polarized Drell-Yan cross section in the $\overline{\mathrm{MS}}$-scheme. The results for the $y$-dependence and the mass distribution agree (after suitable change of the factorization scheme) with earlier results in the literature [25, 26, 27]. We have 
demonstrated that the impact of these corrections on the polarized Drell-Yan cross section is very similar to the impact of the unpolarized corrections. The corrections due to the individual subprocesses turn out to be sizable. However, a $K$-factor between leading and next-to-leading order results can at present not be sensibly predicted due to the uncertainty on the magnitude of the polarized gluon distribution.

The corrections derived in this paper are of particular importance for the extraction of the polarized sea quark distributions from measurements of the Drell-Yan process. As discussed above, a measurement of the asymmetry as function of $x_{F}$ or $y$ can be directly inferred into the $x$-dependence of the polarized sea quark distributions. The results obtained in this paper can be furthermore immediately - keeping in mind the limitations discussed in the introduction - applied to the production of massive vector bosons in polarized proton-proton collisions at RHIC [14].

With the knowledge of the next-to-leading order corrections, it is furthermore possible to quantify the uncertainty of the theoretical prediction and hence the theoretical error on a measurement of the polarized sea quark distributions. We have demonstrated that the absolute value of the Drell-Yan asymmetry in the central region varies by less than 0.01 under variation of the mass factorization scale, demonstrating the perturbative stability of the prediction. This uncertainty is far smaller than the deviation between predictions obtained for different parameterizations of the polarized sea quark distributions.

\section{A Identities for "+"-functions}

The $\epsilon$-expansion of the singular terms in the parton level cross sections of Section 2 yields

$$
\begin{aligned}
(1-z)^{-1-\epsilon} & =-\frac{1}{\epsilon} \delta(1-z)+\frac{1}{(1-z)_{+}}-\epsilon\left(\frac{\ln (1-z)}{1-z}\right)_{+}, \\
z_{1}^{-1-\epsilon} & =-\frac{1}{\epsilon} \delta\left(z_{1}\right)+\frac{1}{\left(z_{1}\right)_{+}}-\epsilon\left(\frac{\ln z_{1}}{z_{1}}\right)_{+} \\
\left(1-z_{1}\right)^{-1-\epsilon} & =-\frac{1}{\epsilon} \delta\left(1-z_{1}\right)+\frac{1}{\left(1-z_{1}\right)_{+}}-\epsilon\left(\frac{\ln \left(1-z_{1}\right)}{1-z_{1}}\right)_{+} .
\end{aligned}
$$

Integration over $z_{1}$ yields the following "+"-functions, all defined on the interval $[0 ; 1]$ :

$$
\frac{1}{\left(z_{1}^{*}\right)_{+}}, \quad \frac{1}{\left(1-z_{1}^{*}\right)_{+}},
$$

and $\delta$-functions:

$$
\delta\left(z_{1}^{*}\right), \quad \delta\left(1-z_{1}^{*}\right) .
$$

Furthermore, various products of the above terms appear. By construction, we have $z=\left(x_{1}^{0} x_{2}^{0}\right) /\left(x_{1} x_{2}\right)$ while $z_{1}^{*}$ is fixed by the definition of $x_{F}$ or $y$ respectively. As the parton level cross section is always integrated over the parton momentum fractions $x_{1}$ and $x_{2}$, it is desirable to re-express the above functions in terms of

$$
\delta\left(x_{1}-x_{1}^{0}\right), \quad \delta\left(x_{2}-x_{2}^{0}\right)
$$


and

$$
\begin{aligned}
& \frac{1}{\left(x_{i}-x_{i}^{0}\right)_{+}} \text {with } \int_{x_{i}^{0}}^{1} \mathrm{~d} x_{i} \frac{f\left(x_{i}\right)}{\left(x_{i}-x_{i}^{0}\right)_{+}} \equiv \int_{x_{i}^{0}}^{1} \mathrm{~d} x_{i} \frac{f\left(x_{i}\right)-f\left(x_{i}^{0}\right)}{x_{i}-x_{i}^{0}}, \\
& \left(\frac{\ln \left(1-x_{i}^{0} / x_{i}\right)}{x_{i}-x_{i}^{0}}\right)_{+} \text {with } \int_{x_{i}^{0}}^{1} \mathrm{~d} x_{i} f\left(x_{i}\right)\left(\frac{\ln \left(1-x_{i}^{0} / x_{i}\right)}{x_{i}-x_{i}^{0}}\right)_{+} \\
& \equiv \int_{x_{i}^{0}}^{1} \mathrm{~d} x_{i}\left[f\left(x_{i}\right)-f\left(x_{i}^{0}\right)\right] \frac{\ln \left(1-x_{i}^{0} / x_{i}\right)}{x_{i}-x_{i}^{0}}, \\
& \frac{1}{\left[\left(x_{1}-x_{1}^{0}\right)\left(x_{2}-x_{2}^{0}\right)\right]_{+}} \quad \text { with } \int_{x_{1}^{0}}^{1} \mathrm{~d} x_{1} \int_{x_{2}^{0}}^{1} \mathrm{~d} x_{2} \frac{f\left(x_{1}, x_{2}\right)}{\left[\left(x_{1}-x_{1}^{0}\right)\left(x_{2}-x_{2}^{0}\right)\right]_{+}} \\
& \equiv \int_{x_{1}^{0}}^{1} \mathrm{~d} x_{1} \int_{x_{2}^{0}}^{1} \mathrm{~d} x_{2} \frac{f\left(x_{1}, x_{2}\right)-f\left(x_{1}^{0}, x_{2}\right)-f\left(x_{1}, x_{2}^{0}\right)+f\left(x_{1}^{0}, x_{2}^{0}\right)}{\left(x_{1}-x_{1}^{0}\right)\left(x_{2}-x_{2}^{0}\right)} .
\end{aligned}
$$

We first consider the $x_{F}$-distributions. All terms containing $\delta(1-z)$ can be simplified by

$$
\delta(1-z) \delta\left(x_{F}-\left[z_{1}\left(x_{1}+x_{2}\right)(1-z)+z x_{1}-x_{2}\right]\right)=\frac{x_{1}^{0} x_{2}^{0}}{x_{1}^{0}+x_{2}^{0}} \delta\left(x_{1}-x_{1}^{0}\right) \delta\left(x_{2}-x_{2}^{0}\right)
$$

and subsequent integration over $z_{1}$. For the remaining terms, we integrate over $z_{1}$ using the $x_{F}$-fixing $\delta$-function, which yields

$$
z_{1}^{*}=\frac{x_{1}^{0}+x_{2}}{(1-z) x_{2}\left(x_{1}+x_{2}\right)}\left(x_{2}-x_{2}^{0}\right) \quad \text { and } \quad 1-z_{1}^{*}=\frac{x_{1}+x_{2}^{0}}{(1-z) x_{1}\left(x_{1}+x_{2}\right)}\left(x_{1}-x_{1}^{0}\right) .
$$

As the integrations over the parton momenta $x_{1}$ and $x_{2}$ are independent from each other, we can perform them in arbitrary order. For terms containing $\delta\left(z_{1}^{*}\right)$ and $1 /\left(z_{1}^{*}\right)_{+}$ only, we choose to integrate $x_{2}$ first. For terms containing $\delta\left(1-z_{1}^{*}\right)$ and $1 /\left(1-z_{1}^{*}\right)_{+}$only, $x_{1}$ is integrated first. We can then make the following replacements:

$$
\begin{aligned}
\delta\left(z_{1}^{*}\right) & =\frac{(1-z) x_{2}^{0}\left(x_{1}+x_{2}^{0}\right)}{x_{1}^{0}+x_{2}^{0}} \delta\left(x_{2}-x_{2}^{0}\right), \\
\frac{1}{\left(z_{1}^{*}\right)_{+}} & =\frac{(1-z) x_{2}\left(x_{1}+x_{2}\right)}{x_{1}^{0}+x_{2}}\left[\frac{1}{\left(x_{2}-x_{2}^{0}\right)_{+}}+\delta\left(x_{2}-x_{2}^{0}\right) \ln \frac{\left(x_{1}^{0}+x_{2}^{0}\right)\left(1-x_{2}^{0}\right) x_{1}}{x_{2}^{0}\left(x_{1}+x_{2}^{0}\right)\left(x_{1}-x_{1}^{0}\right)}\right], \\
\delta\left(1-z_{1}^{*}\right) & =\frac{(1-z) x_{1}^{0}\left(x_{1}^{0}+x_{2}\right)}{x_{1}^{0}+x_{2}^{0}} \delta\left(x_{1}-x_{1}^{0}\right), \\
\frac{1}{\left(1-z_{1}^{*}\right)_{+}} & =\frac{(1-z) x_{1}\left(x_{1}+x_{2}\right)}{x_{1}+x_{2}^{0}}\left[\frac{1}{\left(x_{1}-x_{1}^{0}\right)_{+}}+\delta\left(x_{1}-x_{1}^{0}\right) \ln \frac{\left(x_{1}^{0}+x_{2}^{0}\right)\left(1-x_{1}^{0}\right) x_{2}}{x_{1}^{0}\left(x_{1}^{0}+x_{2}\right)\left(x_{2}-x_{2}^{0}\right)}\right] .
\end{aligned}
$$

The above identities for $\delta\left(z_{1}^{*}\right)$ and $\delta\left(1-z_{1}^{*}\right)$ can be safely applied to terms containing "+"-functions in $(1-z)$. These can then be rewritten as

$$
\delta\left(x_{1}-x_{1}^{0}\right) \frac{1}{(1-z)_{+}}=\delta\left(x_{1}-x_{1}^{0}\right)\left[\frac{x_{2}}{\left(x_{2}-x_{2}^{0}\right)_{+}}+x_{2}^{0} \ln \frac{1-x_{2}^{0}}{x_{2}^{0}} \delta\left(x_{2}-x_{2}^{0}\right)\right],
$$




$$
\begin{aligned}
\delta\left(x_{1}-x_{1}^{0}\right)\left(\frac{\ln (1-z)}{1-z}\right)_{+}=\delta\left(x_{1}-x_{1}^{0}\right) & {\left[x_{2}\left(\frac{\ln \left(1-x_{2}^{0} / x_{2}\right)}{x_{2}-x_{2}^{0}}\right)_{+}\right.} \\
& \left.+x_{2}^{0}\left(\operatorname{Li}_{2}\left(x_{2}^{0}\right)-\frac{\pi^{2}}{6}+\frac{1}{2} \ln ^{2}\left(1-x_{2}^{0}\right)\right) \delta\left(x_{2}-x_{2}^{0}\right)\right]
\end{aligned}
$$

and similar for $(1 \leftrightarrow 2)$. These identities are however inapplicable to the only remaining term

$$
\frac{1}{(1-z)_{+}}\left[\frac{1}{\left(z_{1}^{*}\right)_{+}}+\frac{1}{\left(1-z_{1}^{*}\right)_{+}}\right] \frac{1}{(1-z)\left(x_{1}+x_{2}\right)},
$$

as $\ln \left(x_{1}-x_{1}^{0}\right)$ and $\ln \left(x_{2}-x_{2}^{0}\right)$ appearing in (A.4) are not defined for $z=1$. To overcome this problem, we rewrite

$$
\ln \left(x_{1}-x_{1}^{0}\right)=\ln \frac{x_{1}-x_{1}^{0} x_{2}^{0}}{x_{2}^{0}}-\int_{x_{2}^{0}}^{1} \mathrm{~d} \alpha_{2} \frac{1}{\alpha_{2}-x_{1}^{0} x_{2}^{0} / x_{1}},
$$

and similar for $(1 \leftrightarrow 2)$. After subsequent algebraic manipulations and integration over $\alpha_{1}$ and $\alpha_{2}$, we obtain:

$$
\begin{aligned}
& \frac{1}{(1-z)_{+}}\left[\frac{1}{\left(z_{1}^{*}\right)_{+}}+\frac{1}{\left(1-z_{1}^{*}\right)_{+}}\right] \frac{1}{(1-z)\left(x_{1}+x_{2}\right)}= \\
& \delta\left(x_{1}-x_{1}^{0}\right) \delta\left(x_{2}-x_{2}^{0}\right) \frac{x_{1}^{0} x_{2}^{0}}{x_{1}^{0}+x_{2}^{0}}\left[\ln \frac{x_{1}^{0}}{1-x_{1}^{0}} \ln \frac{x_{2}^{0}}{1-x_{2}^{0}}-\operatorname{Li}_{2}\left(x_{1}^{0}\right)-\frac{1}{2} \ln ^{2}\left(1-x_{1}^{0}\right)\right. \\
& \left.\quad-\operatorname{Li}_{2}\left(x_{2}^{0}\right)-\frac{1}{2} \ln ^{2}\left(1-x_{2}^{0}\right)+\frac{\pi^{2}}{2}\right] \\
& +\delta\left(x_{1}-x_{1}^{0}\right) \frac{x_{1}^{0} x_{2}}{x_{1}^{0}+x_{2}^{0}}\left[\frac{1}{\left(x_{2}-x_{2}^{0}\right)_{+}} \ln \frac{\left(x_{1}^{0}+x_{2}^{0}\right)\left(1-x_{1}^{0}\right)}{x_{1}^{0}\left(x_{1}^{0}+x_{2}\right)}-\left(\frac{\ln \left(1-x_{2}^{0} / x_{2}\right)}{x_{2}-x_{2}^{0}}\right)_{+}\right] \\
& +\delta\left(x_{2}-x_{2}^{0}\right) \frac{x_{1} x_{2}^{0}}{x_{1}^{0}+x_{2}^{0}}\left[\frac{1}{\left(x_{1}-x_{1}^{0}\right)_{+}} \ln \frac{\left(x_{1}^{0}+x_{2}^{0}\right)\left(1-x_{2}^{0}\right)}{x_{2}^{0}\left(x_{1}+x_{2}^{0}\right)}-\left(\frac{\ln \left(1-x_{1}^{0} / x_{1}\right)}{x_{1}-x_{1}^{0}}\right)_{+}\right] \\
& +\frac{\left(x_{1}+x_{2}\right) x_{1} x_{2}}{\left(x_{1}^{0}+x_{2}\right)\left(x_{1}+x_{2}^{0}\right)} \frac{1}{\left[\left(x_{1}-x_{1}^{0}\right)\left(x_{2}-x_{2}^{0}\right)\right]_{+}}
\end{aligned}
$$

Analogous relations can be obtained for the $y$-distributions, where we can first make the replacement

$$
\delta(1-z) \delta\left(y-\frac{1}{2}\left[\ln \frac{x_{1}}{x_{2}}+\ln \frac{z+z_{1}-z z_{1}}{1-z_{1}+z z_{1}}\right]\right)=x_{1}^{0} x_{2}^{0} \delta\left(x_{1}-x_{1}^{0}\right) \delta\left(x_{2}-x_{2}^{0}\right) .
$$

Evaluation of the $z_{1}$-integral in the remaining terms yields $z_{1}^{*}=\frac{x_{1}^{0}\left(x_{2}+x_{2}^{0}\right)}{x_{2}(1-z)\left(x_{1} x_{2}^{0}+x_{1}^{0} x_{2}\right)}\left(x_{2}-x_{2}^{0}\right) \quad$ and $\quad 1-z_{1}^{*}=\frac{x_{2}^{0}\left(x_{1}+x_{1}^{0}\right)}{x_{1}(1-z)\left(x_{1} x_{2}^{0}+x_{1}^{0} x_{2}\right)}\left(x_{1}-x_{1}^{0}\right)$.

Only the identities (A.5) are unchanged for the $y$-distributions, the remaining identities read:

$$
\delta\left(z_{1}^{*}\right)=\frac{(1-z)(1+z) x_{2}^{0}}{2 z} \delta\left(x_{2}-x_{2}^{0}\right),
$$




$$
\begin{aligned}
\frac{1}{\left(z_{1}^{*}\right)_{+}} & =\frac{(1-z) x_{2}\left(x_{1} x_{2}^{0}+x_{1}^{0} x_{2}\right)}{x_{1}^{0}\left(x_{2}+x_{2}^{0}\right)}\left[\frac{1}{\left(x_{2}-x_{2}^{0}\right)_{+}}+\delta\left(x_{2}-x_{2}^{0}\right) \ln \frac{2 x_{1} x_{1}^{0}\left(1-x_{2}^{0}\right)}{x_{2}^{0}\left(x_{1}+x_{1}^{0}\right)\left(x_{1}-x_{1}^{0}\right)}\right], \\
\delta\left(1-z_{1}^{*}\right) & =\frac{(1-z)(1+z) x_{1}^{0}}{2 z} \delta\left(x_{1}-x_{1}^{0}\right), \\
\frac{1}{\left(1-z_{1}^{*}\right)_{+}} & =\frac{(1-z) x_{1}\left(x_{1} x_{2}^{0}+x_{1}^{0} x_{2}\right)}{x_{2}^{0}\left(x_{1}+x_{1}^{0}\right)}\left[\frac{1}{\left(x_{1}-x_{1}^{0}\right)_{+}}+\delta\left(x_{1}-x_{1}^{0}\right) \ln \frac{2 x_{2} x_{2}^{0}\left(1-x_{1}^{0}\right)}{x_{1}^{0}\left(x_{2}+x_{2}^{0}\right)\left(x_{2}-x_{2}^{0}\right)}\right],
\end{aligned}
$$

and

$$
\begin{aligned}
& \frac{1}{(1-z)_{+}}\left[\frac{1}{\left(z_{1}^{*}\right)_{+}}+\frac{1}{\left(1-z_{1}^{*}\right)_{+}}\right] \frac{1}{1-z}= \\
& \delta\left(x_{1}-x_{1}^{0}\right) \delta\left(x_{2}-x_{2}^{0}\right) x_{1}^{0} x_{2}^{0}\left[\ln \frac{x_{1}^{0}}{1-x_{1}^{0}} \ln \frac{x_{2}^{0}}{1-x_{2}^{0}}-\operatorname{Li}_{2}\left(x_{1}^{0}\right)-\frac{1}{2} \ln ^{2}\left(1-x_{1}^{0}\right)\right. \\
& \left.\quad-\operatorname{Li}_{2}\left(x_{2}^{0}\right)-\frac{1}{2} \ln ^{2}\left(1-x_{2}^{0}\right)+\frac{\pi^{2}}{2}\right] \\
& +\delta\left(x_{1}-x_{1}^{0}\right) \frac{x_{1}^{0} x_{2}\left(x_{2}+x_{2}^{0}\right)}{2 x_{2}^{0}}\left[\frac{1}{\left(x_{2}-x_{2}^{0}\right)_{+}} \ln \frac{2 x_{2}^{0}\left(1-x_{1}^{0}\right)}{x_{1}^{0}\left(x_{2}+x_{2}^{0}\right)}-\left(\frac{\ln \left(1-x_{2}^{0} / x_{2}\right)}{x_{2}-x_{2}^{0}}\right)_{+}\right] \\
& +\delta\left(x_{2}-x_{2}^{0}\right) \frac{x_{1} x_{2}^{0}\left(x_{1}+x_{1}^{0}\right)}{2 x_{1}^{0}}\left[\frac{1}{\left(x_{1}-x_{1}^{0}\right)_{+}} \ln \frac{2 x_{1}^{0}\left(1-x_{2}^{0}\right)}{x_{2}^{0}\left(x_{1}+x_{1}^{0}\right)}-\left(\frac{\ln \left(1-x_{1}^{0} / x_{1}\right)}{x_{1}-x_{1}^{0}}\right)_{+}\right] \\
& +\frac{\left(x_{1} x_{2}^{0}+x_{1}^{0} x_{2}\right)^{2} x_{1} x_{2}}{x_{1}^{0} x_{2}^{0}\left(x_{1}+x_{1}^{0}\right)\left(x_{2}+x_{2}^{0}\right)} \frac{1}{\left[\left(x_{1}-x_{1}^{0}\right)\left(x_{2}-x_{2}^{0}\right)\right]_{+}}
\end{aligned}
$$

\section{References}

[1] S.D. Drell and T.M. Yan, Phys. Rev. Lett. 25 (1970) 316; Ann. Phys. 66 (1971) 578.

[2] W.J. Stirling and M.R. Whalley, J. Phys. G19 (1993) D1.

[3] A.D. Martin, R.G. Roberts and W.J. Stirling, Phys. Lett. B354 (1995) 155.

[4] M. Glück, E. Reya and A. Vogt, Z. Phys. C67 (1995) 433.

[5] P. Sutton, A.D. Martin, R.G. Roberts and W.J. Stirling, Phys. Rev. D45 (1992) 2349.

[6] S.D. Ellis and W.J. Stirling, Phys. Lett. B256 (1991) 258.

[7] NA51 collaboration: A. Baldit et al., Phys. Lett. B332 (1994) 244.

[8] SLAC-Yale collaboration: M.J. Alguard et al., Phys. Rev. Lett. 37 (1976) 1261; G. Baum et al., Phys. Rev. Lett. 45 (1980) 2000; 51 (1983) 1135.

EMC collaboration: J. Ashman et al., Nucl. Phys. B328 (1989) 1.

SLAC-E142 collaboration: P.L. Anthony et al., Phys. Rev. Lett. 71 (1993) 959; Phys. Rev. D54 (1996) 6620. 
SMC collaboration: B. Adeva et al., Phys. Lett. B302 (1993) 533; B369 (1996) 93; D. Adams et al., Phys. Lett. B329 (1994) 399; B336 (1994) 125; B357 (1995) 248. SLAC-E143 collaboration: K. Abe et al., Phys. Rev. Lett. 74 (1995) 346; 75 (1995) 25; 76 (1996) 587; Phys. Lett. B364 (1995) 61; preprint SLAC-PUB-96-7242 hepex/9701004).

[9] T. Gehrmann and W.J. Stirling, Phys. Rev. D53 (1996) 6100.

[10] M. Glück, E. Reya, M. Stratmann and W. Vogelsang, Phys. Rev. D53 (1996) 4775.

[11] R.D. Ball, S. Forte and G. Ridolfi, Phys. Lett. B378 (1996) 255.

[12] M. Anselmino, P. Gambino and J. Kalinowski, Torino preprint DFTT-44-96 hepph/9607427).

M. Maul and A. Schäfer, Phys. Lett. B390 (1997) 437.

M. Anselmino et al., Proceedings of the workshop "Future Physics at HERA", 1995/96, eds. G. Ingelman, A. De Roeck and G. Klanner, DESY (Hamburg, 1996), p.827.

[13] S. Gupta, D. Indumathi and M.V.N. Murthy, Z. Phys. C42 (1989) 493, Erratum C44 (1989) 356.

H.Y. Cheng and S.N. Lai, Phys. Rev. D41 (1990) 91.

P. Mathews and R. Ramachandran, Z. Phys. C53 (1992) 305.

P. Mathews and V. Ravindran, Mod. Phys. Lett. A7 (1992) 2695.

T. Gehrmann and W.J. Stirling, Proceedings of the workshop "Future Physics at HERA", 1995/96, eds. G. Ingelman, A. De Roeck and G. Klanner, DESY (Hamburg, 1996), p.847.

[14] RHIC-SPIN collaboration, proposal, 1992.

[15] M. Anselmino et al., Proceedings of the workshop "Future Physics at HERA", 1995/96, eds. G. Ingelman, A. De Roeck and G. Klanner, DESY (Hamburg, 1996), p.837.

V.A. Korotkov and W.D. Nowak, DESY preprint DESY-97-004 (hep-ph/9701371).

[16] G. Altarelli, R.K. Ellis and G. Martinelli, Nucl. Phys. B143 (1978) 521, Erratum B146 (1978) 544.

J. Abad and B. Humpert, Phys. Lett. 80B (1979) 286.

J. Kubar-André and F.E. Paige, Phys. Rev. D19 (1979) 221.

B. Humpert and W.L. van Neerven, Phys. Lett. 84B (1979) 327, Erratum 85B (1979) 471; 89B (1979) 69; Nucl. Phys. B184 (1981) 225.

[17] A.P. Contogouris and J. Kripfganz, Phys. Rev. D20 (1979) 2295.

A.N. Schellekens and W.L. van Neerven, Phys. Rev. D21 (1980) 2619; D22 (1980) 1623.

T. Matsuura and W.L. van Neerven, Z. Phys. C38 (1988) 623.

T. Matsuura, S.C. van der Marck and W.L. van Neerven, Phys. Lett. B211 (1988) 
171; Nucl. Phys. B319 (1989) 570.

R. Hamberg, T. Matsuura and W.L. van Neerven, Nucl. Phys. B345 (1990) 331; B359 (1991) 343.

W.L. van Neerven and E.B. Zijlstra, Nucl. Phys. B382 (1992) 11.

[18] G. Altarelli, R.K. Ellis and G. Martinelli, Nucl. Phys. B157 (1979) 461.

[19] J. Kubar, M. le Bellac, J.L. Meunier and G. Plaut, Nucl. Phys. B175 (1980) 251.

[20] P.J. Rijken and W.L. van Neerven, Phys. Rev. D51 (1995) 44.

[21] C.T.H. Davies and W.J. Stirling, Nucl. Phys. B244 (1984) 337.

C.T.H. Davies, W.J. Stirling and B.R. Webber, Nucl. Phys. B256 (1985) 413.

G. Altarelli, R.K. Ellis, M. Greco and G. Martinelli, Nucl. Phys. B246 (1984) 12.

G. Altarelli, R.K. Ellis and G. Martinelli, Z. Phys. C27 (1985) 617.

[22] P. Arnold and M.H. Reno, Nucl. Phys. B319 (1989) 37.

R.J. Gonsalves, J. Pawlowski and C.F. Wai, Phys. Rev. D40 (1989) 2245.

[23] E. Mirkes, J.G. Körner and G.A. Schuler, Phys. Lett. B259 (1991) 151.

[24] H. Baer and M.H. Reno, Phys. Rev. D43 (1991) 2892.

W.T. Giele, E.W.N. Glover and D.A. Kosower, Nucl. Phys. B403 (1993) 633.

[25] P. Ratcliffe, Nucl. Phys. B223 (1982) 45.

[26] A. Weber, Nucl. Phys. B382 (1992) 63.

[27] B. Kamal, Phys. Rev. D53 (1996) 1142.

[28] W. Vogelsang and A. Weber, Phys. Rev. D48 (1993) 2073.

A.P. Contogouris, B. Kamal and Z. Merebashvili, Phys. Lett. B337 (1994) 169.

[29] R. Mertig and W.L. van Neerven, Z. Phys. C70 (1996) 637.

W. Vogelsang, Phys. Rev. D54 (1996) 2023; Nucl. Phys. B475 (1996) 47.

[30] G. Altarelli and G.G. Ross, Phys. Lett. B212 (1988) 391.

R.D. Carlitz, J.C. Collins and A.H. Mueller, Phys. Lett. B214 (1988) 229.

G. Altarelli and B. Lampe, Z. Phys. C47 (1990) 315.

G.T. Bodwin and J. Qiu, Phys. Rev. D41 (1990) 2755.

W. Vogelsang, Z. Phys. C53 (1991) 275.

[31] G. t'Hooft and M. Veltman, Nucl. Phys. B44 (1972) 189.

P. Breitenlohner and D. Maison, Comm. Math. Phys. 52 (1977) 11.

[32] S. Larin, Phys. Lett. B303 (1993) 113.

[33] M.A. Ahmed and G.G. Ross, Nucl. Phys. B111 (1976) 441.

G. Altarelli and G. Parisi, Nucl. Phys. B126 (1977) 298.

[34] M. Glück, E. Reya and A. Vogt, Phys. Lett. B285 (1992) 285. 